\title{
Ternary quadratic forms that represent zero: the function field case
}

\author{
by \\ Mireille CAR (Marseille)
}

1. Introduction. Let $K$ be a global function field with field of constants $k$, a finite field with $q$ elements and odd characteristic. Let $S$ be a finite set of $s>0$ places of $K$ and let $R_{S}$ denote the ring of $S$-integers of $K$, that is, the set of $a \in K$ such that $v(a) \geq 0$ for each place $v \notin S$. For $s$-tuples $\mathbf{m}=\left(m_{v}\right)_{v \in S}$ and $\mathbf{n}=\left(n_{v}\right)_{v \in S}$ of rational integers, let $Q_{S}(\mathbf{m}, \mathbf{n})$ denote the number of pairs $(a, b)$ of integers of $R_{S}$ such that $v(a)=m_{v}$ and $v(b)=n_{v}$ for all $v \in S$ and the quadratic form

$$
\left(f_{a, b}\right) \quad X^{2}-a Y^{2}-b Z^{2}
$$

represents 0 over the field $K$. We give an asymptotic estimate for $Q_{S}(\mathbf{m}, \mathbf{n})$ for $s$-tuples $\mathbf{m}$ and $\mathbf{n}$ such that the numbers

$$
\|\mathbf{m}\|=-\sum_{v \in S} f_{v} m_{v}, \quad\|\mathbf{n}\|=-\sum_{v \in S} f_{v} n_{v}
$$

tend to $\infty, f_{v}$ denoting the degree of the place $v$.

The present paper can be viewed as a generalization of [1] where the author dealt with the case of a rational function field. That case was a polynomial analogue of questions asked by Serre [8] and solved by Hooley [5] and Guo [4] about the size of the number $H(x)$ of pairs $(a, b) \in \mathbb{Z}^{2}$ such that $|a|,|b| \leq x$ and the ternary quadratic form

$$
X^{2}+a Y^{2}+b Z^{2}
$$

represents 0 over the field $\mathbb{Q}$. Presently no number field analogue of the theorems proved in what follows is known.

This paper is organized as follows. Notations and statements of the main theorems are given in Section 2. Auxiliary estimates concerning arithmetic functions and character sums are given in the third section. Section 4 is the

2000 Mathematics Subject Classification: Primary 11T55.

Key words and phrases: quadratic forms, function fields. 
main part of the paper. In that section, we require the coefficients $a$ and $b$ to belong to a ring $R_{\left\{v_{0}\right\}}$ since dealing with the case where $S$ reduces to one element $v_{0}$ is easier. In this setting we study a more general problem. This study allows us to get as a corollary an estimate for $Q_{S}(\mathbf{m}, \mathbf{n})$, obtained in the last section.

2. Notations and statement of the results. Let $g$ be the genus of $K$ and let $h$ be its divisor class number, that is, the number of classes of divisors of degree 0 .

Let $V=V(K)$ denote the set of places of $K$. When there is no danger of confusion we denote by the same symbol a place and the normalized discrete valuation associated with it.

The zeta-function of the field $K$ is defined on the open disk $D_{1 / q}$ formed by the complex numbers $z$ such that $|z|<1 / q$ by

$$
\zeta_{K}(u)=\prod_{v \in V}\left(1-u^{f_{v}}\right)^{-1} .
$$

(Since we shall use the $\zeta$-function in its rational form we have chosen to denote it in an unusual way.)

For $s$-tuples $\mathbf{m}=\left(m_{v}\right)_{v \in S}$ and $\mathbf{n}=\left(n_{v}\right)_{v \in S}$ of rational integers, let $Q_{S}(\mathbf{m}, \mathbf{n}), Q_{S}^{\prime}(\mathbf{m}, \mathbf{n})$ and $Q_{1, S}(\mathbf{m}, \mathbf{n})$ denote respectively the number of pairs $(a, b)$ of integers in $R_{S}$, of square-free integers in $R_{S}$, and of square-free coprime integers in $R_{S}$, such that $v(a)=m_{v}$ and $v(b)=n_{v}$ for all $v \in S$, and the quadratic form

$$
\left(f_{a, b}\right) \quad X^{2}-a Y^{2}-b Z^{2}
$$

represents 0 over the field $K$.

We prove the following theorem.

TheOREM 2.1. Let $\lambda$ and $\theta$ be real numbers such that

$$
\frac{3 \log 2}{2 \log q}<\lambda \leq 1 \quad \text { and } \quad \frac{\log 2}{\log q}<\theta \leq 1 .
$$

Let $\mathbf{m}=\left(m_{v}\right)_{v \in S}$ and $\mathbf{n}=\left(n_{v}\right)_{v \in S}$ be s-tuples of rational integers, let $\tau(\mathbf{m}, \mathbf{n})$ be the number of indices $v$ such that $m_{v}$ or $n_{v}$ is odd, and let

$$
\|\mathbf{m}\|=-\sum_{v \in S} f_{v} m_{v}, \quad\|\mathbf{n}\|=-\sum_{v \in S} f_{v} n_{v} .
$$

(i) If $0<\lambda \max (\|\mathbf{m}\|,\|\mathbf{n}\|) \leq \min (\|\mathbf{m}\|,\|\mathbf{n}\|)$, then

$$
Q_{S}(\mathbf{m}, \mathbf{n})=2^{-\tau(\mathbf{m}, \mathbf{n})} C(S) \frac{q^{\|\mathbf{m}\|+\|\mathbf{n}\|}}{\|\mathbf{m}\|^{1 / 2}\|\mathbf{n}\|^{1 / 2}}+O\left(\frac{q^{\|\mathbf{m}\|+\|\mathbf{n}\|}}{\|\mathbf{m}\|\|\mathbf{n}\|}\right),
$$


where

$$
\begin{aligned}
C(S)= & \frac{2 \zeta_{K}\left(q^{-2}\right) q^{1-g}(q-1)}{h \pi} \\
& \times \prod_{v \in S}\left(1-\frac{1}{q^{2 f_{v}}}\right)\left(1-\frac{1}{q^{f_{v}}}\right) \cdot \prod_{\substack{v \in V \\
v \in S}}\left(1+\frac{1}{2 q^{f_{v}}\left(q^{f_{v}}+1\right)}\right)
\end{aligned}
$$

and where the constants involved in the $O$ symbol depend only on $K, S$ and $\lambda$.

(ii) If $0<\theta \max (\|\mathbf{m}\|,\|\mathbf{n}\|) \leq \min (\|\mathbf{m}\|,\|\mathbf{n}\|)$, then

$$
\begin{aligned}
Q_{1, S}(\mathbf{m}, \mathbf{n}) & =2^{-\tau(\mathbf{m}, \mathbf{n})} C_{1}(S) \frac{q^{\|\mathbf{m}\|+\|\mathbf{n}\|}}{\|\mathbf{m}\|^{1 / 2}\|\mathbf{n}\|^{1 / 2}}+O\left(\frac{q^{\|\mathbf{m}\|+\|\mathbf{n}\|}}{\|\mathbf{m}\|\|\mathbf{n}\|}\right), \\
Q_{S}^{\prime}(\mathbf{m}, \mathbf{n}) & =2^{-\tau(\mathbf{m}, \mathbf{n})} C^{\prime}(S) \frac{q^{\|\mathbf{m}\|+\|\mathbf{n}\|}}{\|\mathbf{m}\|^{1 / 2}\|\mathbf{n}\|^{1 / 2}}+O\left(\frac{q^{\|\mathbf{m}\|+\|\mathbf{n}\|}}{\|\mathbf{m}\|\|\mathbf{n}\|}\right),
\end{aligned}
$$

where

$$
\begin{aligned}
& C_{1}(S)=\frac{2 q^{1-g}(q-1)}{\pi h \zeta_{K}\left(q^{-2}\right)} \prod_{v \in S}\left(1+\frac{1}{q^{f_{v}}}\right)^{-1}, \\
& C^{\prime}(S)=\frac{2 q^{1-g}(q-1)}{\pi h \zeta_{K}\left(q^{-2}\right)} \prod_{v \in S}\left(1+q^{-f_{v}}\right)^{-1} \cdot \prod_{\substack{v \in V \\
v \notin S}}\left(1+\frac{1}{2 q^{f_{v}}\left(q^{f_{v}}+1\right)}\right)
\end{aligned}
$$

and where the constants involved in the $O$ symbols depend only on $K, S$ and $\theta$.

Let $v \in V$. Let $K_{v}, K_{v}^{\star}, O_{v}$, and $U_{v}$ denote respectively the completion of the field $K$ at the place $v$, the multiplicative group of the field $K_{v}$, the valuation ring of $K_{v}$, and the group of units of the ring $O_{v}$. Moreover, let $f_{v}$ denote the residual degree of $v$.

For a rational integer $j>0$ let $U_{v}^{(j)}$ denote the subgroup formed by the $u \in U_{v}$ such that $v(u-1) \geq j$. Once for all we choose, for any $v \in V$, a uniformizing element $\pi_{v} \in K$. There is a subfield $k_{v}$ of $K_{v}$ isomorphic to the residual field at $v$ such that every non-zero element $\alpha \in K_{v}$ admits a unique expansion

$$
\alpha=\sum_{j=n}^{\infty} a_{j} \pi_{v}^{j},
$$

with $n=v(\alpha), a_{j} \in k_{v}, a_{n} \neq 0$ [7]. Hence $\alpha$ is uniquely written as a product

$$
\alpha=\operatorname{sgn}_{v}(\alpha) \pi_{v}^{v(\alpha)} u_{v}(\alpha)
$$

with $\operatorname{sgn}_{v}(\alpha) \in k_{v}$ and $u_{v}(\alpha) \in U_{v}^{(1)}$. 
Let $v_{0}$ be a place of $K$ and let $R=R_{\left\{v_{0}\right\}}$. We denote by $\mathcal{I}=\mathcal{I}(R)$ the set of non-zero integral ideals of $R$, by $\mathcal{P}=\mathcal{P}(R)$ the set of prime ideals of $R$, by $\mathcal{F}=\mathcal{F}(R)$ the group of fractional ideals of $R$ in $K$, by $\mathcal{S F}=\mathcal{S F}(R)$ the set of square-free ideals of $R$, by $\operatorname{Pr}=\operatorname{Pr}(R)$ the monoid of non-zero principal ideals of $R$, and by $\mathcal{C} \ell=\mathcal{C} \ell(R)$ the ideal class group of $R$.

The set $V$ is the union of the place $v_{0}$ and the $P$-adic places $v_{P}$ for $P$ running through the set $\mathcal{P}$ of prime ideals of $R$. In order to reduce notation, we set

$$
f_{v_{P}}=f_{P}, \quad K_{v_{P}}=K_{P}, \quad U_{v_{P}}^{j}=U_{P}^{(j)}
$$

for each $P \in \mathcal{P}$ or for $P=0$.

Let $H \in \mathcal{I}(R)$. We say that a fractional ideal $J \in \mathcal{F}(R)$ is coprime to $H$ if $v_{P}(J)=0$ for any prime ideal $P$ dividing $H$. For any subset $\mathcal{E}$ of $\mathcal{F}(R)$, we denote by $\mathcal{E}_{H}$ the set of $Y \in \mathcal{E}$ coprime to $H$.

Let $\widehat{\mathcal{C} \ell}=\widehat{\mathcal{C} \ell(R)}$ be the group of characters of $\mathcal{C} \ell(R)$. Let $\chi \in \widehat{\mathcal{C} \ell}$. The character $\chi^{\star}$ of the group of fractional ideals of $R$ derived from $\chi$ is defined by

$$
\chi^{\star}(Y)=\chi(c \ell(Y))
$$

where $c \ell(Y)$ denotes the class of $Y$ in the ideal class group $C \ell(R)$. In what follows, we shall abuse language and denote by the same symbol $\chi$ the character $\chi \in \widehat{\mathcal{C} \ell}$ and the derived character $\chi^{\star}$.

We set

$$
\varrho(R)=\frac{q^{f_{0}}-1}{q-1} .
$$

The group $\mathcal{F}(R)$ is free, generated by the set $\mathcal{P}(R)$. Thus, the subgroup $\operatorname{FPr}(R)$ formed by the non-zero principal fractional ideals of $R$ in $K$ is free. Let $\mathcal{B}$ be a basis of this free group. For each $B \in \mathcal{B}$, let $b_{B} \in K$ be a generator of $B$ chosen once for all. Then the subgroup $\mathcal{H}$ of $K^{\star}$ generated by $\left\{b_{B} ; B \in \mathcal{B}\right\}$ is isomorphic to $\operatorname{FPr}(R)$. Let $\mathcal{M}$ denote the set $\mathcal{H} \cap R$ of integral elements of $\mathcal{H}$. The set $\mathcal{M}$ is a multiplicative monoid such that every principal ideal in $R$ is generated by a unique element of $\mathcal{M}$. The elements of $\mathcal{M}$ will be called monic. (In the rational case, one can take for $\mathcal{B}$ the set of ideals generated by the monic irreducible polynomials and for $\mathcal{M}$ the set of monic polynomials.) For $A_{1}, \ldots, A_{r} \in \mathcal{I}$, the greatest common divisor of $A_{1}, \ldots, A_{r}$ is denoted by $\left(A_{1}, \ldots, A_{r}\right)$. For any non-zero $H \in \mathcal{I}(R)$, let $\omega(H)$ denote the number of distinct prime divisors of $H$, and $|H|$ the number of elements of the quotient ring $R / H$. Then $|H|$ is a power of $q$. We define the degree $f_{H}$ of the ideal $H$ by

$$
|H|=q^{f_{H}} .
$$

This notation agrees with the notation $f_{P}=f_{v_{P}}$ used for prime ideals. We 
note that

$$
f_{H}=\sum_{\substack{P \in \mathcal{P} \\ P \mid H}} v_{P}(H) f_{P}
$$

and that this notation extends in a natural way to fractional ideals.

The divisor class number $h$ of $K$ and the ideal class number $h_{0}$ of $R$, that is, the order of the ideal class group $\mathcal{C} \ell$, are connected by the identity

$$
h_{0}=h f_{0} \text {. }
$$

See [6] for a proof.

We shall denote by $D_{r}$ the open disk formed by the complex numbers $z$ such that $|z|<r$, and by $z^{1 / 2}$ the branch of $z \mapsto z^{1 / 2}$ for which $1^{1 / 2}=1$.

The following properties of the zeta-function $\zeta_{K}$ are well-known (cf. [9]):

$$
\zeta_{K}(u)=\frac{P_{K}(u)}{(1-u)(1-q u)},
$$

where $P_{K}$ is a polynomial of degree $2 g$.

If $g>0$, there exist algebraic numbers $\varrho_{1}, \ldots, \varrho_{g}$ such that

$$
P_{K}(u)=\prod_{i=1}^{g}\left(1-\varrho_{i} u\right)\left(1-\bar{\varrho}_{i} u\right), \quad\left|\varrho_{i}\right|=\sqrt{q} .
$$

Moreover,

$$
P_{K}(1)=h,
$$

and $P_{K}$ satisfies the functional equation

$$
P_{K}(u)=q^{g} u^{2 g} P_{K}\left(\frac{1}{q u}\right) .
$$

(For the rational function field $K$, we have $g=0, h=1$.)

All constants occurring in this work depend on $K$ and other parameters. We agree that a constant denoted $a\left(x_{1}, \ldots, x_{r}\right)$ depends only on $K, x_{1}, \ldots, x_{r}$, or possibly only on $x_{1}, \ldots, x_{r}$, and that a constant denoted $b$ depends only on $K$, or is absolute. We shall denote by $(a)$ the principal ideal $R a$ and by \#E the cardinality of any finite set $E$. If $H$ and $Y$ are ideals in $\mathcal{I}(R)$ and if $a$ is a non-zero element of $R$, then $H \mid Y$ and $H \mid a$ will mean respectively that the ideal $H$ divides the ideal $Y$ or the principal ideal $(a)$.

3. Auxiliary estimates. In this section we collect all technical estimates required for the proof. Let us introduce some new notations and definitions.

Once for all, we choose an ideal $I_{1} \in \mathcal{F}(R)$ such that $f_{I_{1}}=1$. According to [9, Chap. VII], such an ideal exists. 
REMARK 3.1.

(i) Let $a \in R$. Then

$$
f_{(a)}=-f_{0} v_{0}(a) .
$$

(ii) The map $X \mapsto f_{X}$ is a surjective homomorphism from $\mathcal{F}(R)$ onto $\mathbb{Z}$.

(iii) If $I$ and $J$ are fractional ideals belonging to the same ideal class $\Gamma$, then

$$
f_{I} \equiv f_{J} \bmod f_{0} .
$$

Thus, there is a unique surjective homomorphism $\varphi: \Gamma \mapsto \varphi_{\Gamma}$ of the group $\mathcal{C} \ell=\mathcal{C} \ell(R)$ onto $\mathbb{Z} / \mathbb{Z} f_{0}$ such that

$$
\varphi_{\Gamma}=f_{H} \quad \text { for any } H \in \Gamma .
$$

Proof. See [2, Remarques III.4].

Let $n$ be a non-negative integer and let $\Gamma \in \mathcal{C} \ell$. Let $i(n)$ and $i(\Gamma, n)$ denote respectively the number of ideals $Y \in \mathcal{I}$ or $Y \in \Gamma$ such that $f_{Y}=n$. We write $n \in \varphi_{\Gamma}$, resp. $n \notin \varphi_{\Gamma}$, whenever the congruence classes $n+\mathbb{Z} f_{0}$ and $\varphi_{\Gamma}$ are equal, resp. different.

Proposition 3.2. Let $n$ be a non-negative integer and let $\Gamma \in \mathcal{C} \ell$. Then

$$
\begin{gathered}
i(\Gamma, n)=0 \quad \text { if } n \notin \varphi_{\Gamma}, \\
i(\Gamma, n) \leq \varrho(R) q^{n}, \quad i(n) \leq h \varrho(R) q^{n}, \\
i(\Gamma, n)=\varrho(R) q^{n+1-g-f_{0}} \quad \text { if } n \in \varphi_{\Gamma} \text { and } n \geq 2 g-1+f_{0}, \\
i(n)=h \varrho(R) q^{n+1-g-f_{0}} \quad \text { if } n \geq 2 g-1+f_{0} .
\end{gathered}
$$

Proof. We get (3.4) from (3.2) and (3.3). If $\gamma$ is the unit class then $i(\gamma, 0)=i(0)=1$. Suppose $n>0$. By (3.2), (3.3), and (3.4),

$$
i(n)=\sum_{\Gamma \in \mathcal{C} \ell} i(\Gamma, n)=\sum_{\substack{\Gamma \in \mathcal{C} \ell \\ n \in \varphi_{\Gamma}}} i(\Gamma, n) .
$$

Since there are exactly $h_{0} / f_{0}=h$ ideal classes $\Gamma$ such that $n \in \varphi_{\Gamma}$, it suffices to prove the first part of (3.5) and (3.6) in the case where $n \in \varphi_{\Gamma}$. Let $H \in \mathcal{I}(R)$ belong to the class $\Gamma^{-1}$. (Such an ideal exists, since if $J \in \mathcal{F}(R)$, then there exists a non-zero element $x \in R$ such that $x J \in \mathcal{I}(R)$, and the ideals $J$ and $x J$ belong to the same class.) Then $n+f_{H} \equiv 0 \bmod f_{0}$. Let $m$ be defined by

$$
n+f_{H}=m f_{0} .
$$

Let $Y \in \mathcal{I}(R)$. Then $Y \in \Gamma$ if and only if there exists $y \in \mathcal{M}$, necessarily unique, such that $Y H=(y)$, and in this case, by the product formula, $f_{Y}=n$ if and only if $-f_{0} v_{0}(y)=n+f_{H}$. Since the group of units of 
$R=R_{v_{0}}$ is the multiplicative group $k^{\star}$,

$$
(q-1) i(\Gamma, n)=\#\left\{y \in R ; v_{0}(y)=-m, H \mid y\right\} .
$$

For $r \in\{m, m-1\}$, consider the divisor

$$
A(r, H)=r v_{0}-\sum_{\substack{P \in \mathcal{P} \\ P \mid H}} v_{P}(H) v_{P}
$$

and the set $\Lambda(A(r, H))$ formed by the $y \in K$ such that $v(y) \geq-v(A(r, H))$ for any $v \in V(K)$. Then $\Lambda(A(r, H))$ is a $k$-vector space of finite dimension over $k$. The Riemann-Roch theorem [3] connects the dimension $\lambda_{r}$ of $\Lambda(A(r, H))$ and the degree

$$
F_{A(r, H)}=r f_{0}-\sum_{\substack{P \in \mathcal{P} \\ P \mid H}} f_{P} v_{P}(H)
$$

of the divisor $A(r, H)$. One has

$$
\begin{gathered}
\lambda_{r} \leq \max \left(0,1+F_{A(r, H)}\right) \leq n, \\
\lambda_{m}-\lambda_{m-1} \leq F_{A(m, H)}-F_{A(m-1, H)}=f_{0},
\end{gathered}
$$

and if $F_{A(r, H)} \geq 2 g-1$, then

$$
\lambda_{r}=F_{A(r, H)}+1-g .
$$

By (2),

$$
(q-1) i(\Gamma, n)=\# \Lambda(A(m, H))-\# \Lambda(A(m-1, H)) .
$$

If $\Lambda(A(m, H))=\Lambda(A(m-1, H))$, then by $(7), i(\Gamma, n)=0$ and (3.5) is proved. If $\Lambda(A(m, H)) \neq \Lambda(A(m-1, H))$, the quotient $\Lambda(A(m, H)) / \Lambda(A(m-1, H))$ has $q^{f_{0}}-1$ non-zero elements, hence, by (7) and (2.4),

$$
i(\Gamma, n)=\varrho(R) \# \Lambda(A(m-1, H)),
$$

and by (4),

$$
i(\Gamma, n) \leq \varrho(R) q^{n} .
$$

Now, suppose $n \geq 2 g-1+f_{0}$. Then, by (6),

$$
i(\Gamma, n)=\varrho(R) q^{n+1-g-f_{0}},
$$

proving (3.6).

Proposition 3.3. Let $\chi$ be a character of $\mathcal{C} \ell(R)$. Then the series

$$
L(\chi, u)=\sum_{Y \in \mathcal{I}} \chi(Y) u^{f_{Y}}
$$

is absolutely convergent in the disk $D_{1 / q}$, and for $u \in D_{1 / q}$,

$$
L(\chi, u)=\prod_{P \in \mathcal{P}}\left(1-\chi(P) u^{f_{P}}\right)^{-1} .
$$


Moreover,

(i) if $\chi$ is trivial on $\operatorname{Ker} \varphi$ (see Remark 3.1(iii)), then

$$
L(\chi, u)=\zeta_{K}\left(\chi\left(I_{1}\right) u\right)\left(1-u^{f_{0}}\right),
$$

(ii) if $\chi$ is not trivial on $\operatorname{Ker} \varphi$, then $L(\chi, u)$ is a polynomial of degree $2 g-2+f_{0}$ and

$$
L(\chi, u)=\prod_{i=1}^{2 g-2+f_{0}}\left(1-\omega_{i} u\right),
$$

with $\left|\omega_{i}\right|=q^{1 / 2}$ for $1 \leq i \leq 2 g-2$ and $\left|\omega_{i}\right|=1$ for $2 g-1 \leq i \leq$ $2 g-2+f_{0}$.

Proof. See [2, Proposition III.5].

Proposition 3.4. Let $a>1$ be a real number. Then

$$
\begin{aligned}
& \prod_{P \in \mathcal{P}}\left(1-|P|^{-a}\right)=\frac{\left(1-q^{-a}\right)\left(1-q^{1-a}\right)}{\left(1-q^{-f_{0} a}\right) P_{K}\left(q^{-a}\right)}, \\
& \prod_{P \in \mathcal{P}}\left(1+|P|^{-a}\right)=\frac{P_{K}\left(q^{-a}\right)\left(1-q^{1-2 a}\right)\left(1+q^{-a}\right)}{P_{K}\left(q^{-2 a}\right)\left(1-q^{1-a}\right)\left(1+q^{f_{0} a}\right)} .
\end{aligned}
$$

Proof. Let $u \in D_{1 / q}$. By (2.1) and (2.8),

$$
\prod_{P \in \mathcal{P}}\left(1-u^{f_{P}}\right)^{-1}=\frac{\left(1-u^{f_{0}}\right) P_{K}(u)}{(1-u)(1-q u)} .
$$

We get (3.12) and (3.13) by taking $u=q^{-a}$ and $u=q^{-2 a}$.

Let $l \geq 2$ be an integer and let $\mu_{l}$ denote the group of $l$ th roots of unity.

Proposition 3.5. Let $\chi \in \widehat{\mathcal{C} \ell(R)}$. Let $\Psi$ be a morphism from the group $\mathcal{F}(R)$ of fractional ideals to $\mu_{l}$ and let $H \in \mathcal{I}(R)$. Then the series

$$
L(\chi \Psi, u)=\sum_{Y \in \mathcal{I}_{H}} \chi(Y) \Psi(Y) u^{f_{Y}}
$$

is absolutely convergent in the disk $D_{1 / q}$, and for $u \in D_{1 / q}$,

$$
L(\chi \Psi, u)=\prod_{P \in \mathcal{P}_{H}}\left(1-\chi(P) \Psi(P) u^{f_{P}}\right)^{-1} .
$$

Moreover, if $\Psi$ is not trivial on the group $\operatorname{FPr}(R)$ of principal fractional ideals and for any $x \in K$,

$$
x \in U_{0}^{(1)} \text { and } x \equiv 1 \bmod H \Rightarrow \Psi((x))=1,
$$


then $L(\chi \Psi, u)$ is a polynomial of degree $d(\chi \Psi) \leq 2 g-2+f_{0}+f_{H}$ and

$$
L(\chi \Psi, u)=\prod_{i=1}^{d(\chi \Psi)}\left(1+\varrho_{i} u\right)
$$

with $\left|\varrho_{i}\right| \in\left\{q^{1 / 2}, 1\right\}$.

Proof. See [2, Proposition III.6].

Proposition 3.6. Let $\chi \in \widehat{\mathcal{C} \ell(R)}$. Let $\Psi$ be a morphism from the group $\mathcal{F}(R)$ to $\mu_{l}$ and let $H \in \mathcal{I}(R)$ satisfy (3.16). Let $A \in \mathcal{I}(R)$ be coprime to $H$ and for any non-negative integer $n$, let

$$
a(\chi, \Psi, H, A, n)=\sum_{\substack{Y \in \mathcal{S F}_{H A} \\ f_{Y}=n}} \chi(Y) \Psi(Y) 2^{-\omega(Y)} .
$$

Then the series

$$
f(z)=\sum_{n=0}^{\infty} a(\chi, \Psi, H, A, n)\left(\frac{z}{q}\right)^{n}=\sum_{Y \in \mathcal{S F}_{H A}} \chi(Y) \Psi(Y) 2^{-\omega(Y)}\left(\frac{z}{q}\right)^{f_{Y}}
$$

is absolutely convergent in the open disk $D_{1}$, the product

$$
G(z)=\prod_{P \in \mathcal{P}}\left(1-\frac{3}{4}\left(\chi(P) \Psi(P)\left(\frac{z}{q}\right)^{f_{P}}\right)^{2}-\frac{1}{4}\left(\chi(P) \Psi(P)\left(\frac{z}{q}\right)^{f_{P}}\right)^{3}\right)
$$

is absolutely convergent in the open disk $D_{\sqrt{q}}$, and for $z \in D_{1}$ we have

$$
\left(\frac{f(z)}{U(z)}\right)^{2}=L\left(\chi \Psi, \frac{z}{q}\right) G(z)
$$

where

$$
U(z)=\prod_{\substack{P \in \mathcal{P} \\ P \mid A H}}\left(1+\frac{1}{2} \chi(P) \Psi(P)\left(\frac{z}{q}\right)^{f_{P}}\right)^{-1} .
$$

Moreover, if one of the following two hypotheses is satisfied:

(i) there exists $x \in \mathcal{M}$ with $\Psi((x)) \neq 1$,

(ii) $H=(1), \Psi$ is trivial on $\mathcal{F}(R)$ and $\chi$ is not trivial on $\operatorname{Ker} \varphi$, then for any $n \geq 1$,

$$
|a(\chi, \Psi, H, A, n)| \leq \alpha_{1}(R) 2^{f_{H} / 2} 2^{\omega(A H)} n^{1 / 2} q^{n / 2}
$$

with $\alpha_{1}(R)$ a constant.

Proof. With the necessary adaptations the proof follows the proof of Proposition 2.2 in [1]. 
Corollary 3.7. Let $H \in \mathcal{I}(R)$ and let $\Psi$ be a morphism from the group $\mathcal{F}(R)_{H}$ to the group $\mu_{l}$ non-trivial on the subgroup of principal ideals and satisfying (3.16). Let $A \in \mathcal{I}(R)$ and let $B \in \mathcal{I}(R)$ be coprime to $H$. For any non-negative integer $n$, let

$$
b(\Psi, H, A, B, n)=\sum_{\substack{Y \in \mathcal{S F}_{B H} \\ f_{Y}=n \\ A Y \in \operatorname{Pr}}} \Psi(Y) 2^{-\omega(Y)} .
$$

Then

$$
|b(\chi, \Psi, H, A, n)| \leq \alpha_{1}(R) 2^{f_{H} / 2} 2^{\omega(B H)} n^{1 / 2} q^{n / 2} .
$$

Proof. By orthogonality,

$$
(\# \mathcal{C} \ell) b(\Psi, H, A, B, n)=\sum_{\chi \in \widehat{\mathcal{C} \ell}} \chi(A) u(\chi, n),
$$

where for any integer $j \geq 0$,

$$
u(\chi, j)=\sum_{\substack{Y \in \mathcal{S F}_{H} \\ f_{Y}=j}} \chi(Y) \Psi(Y) 2^{-\omega(Y)} .
$$

The sum $u(\chi, j)$ is the sum $a(\chi, \Psi, H, B, j)$ defined by (3.18). Then, by (3.23),

$$
|u(\chi, n)| \leq \alpha_{1}(R) 2^{f_{H} / 2} 2^{\omega(B H)} n^{1 / 2} q^{n / 2},
$$

proving (3.25).

Proposition 3.8. Let $\eta \in] 0,1 / 2[$. Then, for $J, A \in \mathcal{I}(R)$ and any positive integer $n \equiv-f_{A} \bmod f_{0}$,

$$
\begin{aligned}
& \left|\sum_{\substack{Y \in \mathcal{S F}_{J} \\
f_{Y}=n \\
A Y \in \operatorname{Pr}}} 2^{-\omega(Y)}-B_{1}(R) \Omega(J) q^{n} n^{-1 / 2}\right| \\
& \quad \leq \alpha_{2}(R) 2^{\omega(J)} q^{n / 2} n^{1 / 2}+\alpha_{3}(R, \eta) \lambda_{\eta}(J) q^{n} n^{-3 / 2}
\end{aligned}
$$

with

$$
\begin{aligned}
B_{1}(R) & =\left(\frac{h \varrho(R)}{\pi h q^{g+f_{0}-1}}\right)^{1 / 2} \prod_{P \in \mathcal{P}}\left(1+\frac{1}{2|P|}\right)\left(1-\frac{1}{|P|}\right)^{1 / 2}, \\
\Omega(J) & =\prod_{\substack{P \in \mathcal{P} \\
P \mid J}}\left(1+\frac{1}{2|P|}\right)^{-1}, \\
\lambda_{\eta}(J) & =\prod_{\substack{P \in \mathcal{P} \\
P \mid J}}\left(1-\frac{|P|^{\eta-1}}{2}\right)^{-1},
\end{aligned}
$$

$\alpha_{2}(R)$ and $\alpha_{3}(R, \eta)$ being constants. 
Proof. Let $n>0$ be a rational integer and let

$$
x_{n}=\sum_{\substack{Y \in \mathcal{S F}_{J} \\ A Y \in \operatorname{Pr} \\ f_{Y}=n}} 2^{-\omega(Y)} .
$$

By orthogonality,

$$
h_{0} x_{n}=\sum_{\chi \in \widehat{C \ell \ell}} \chi(A) u(\chi, n)
$$

where

$$
u(\chi, k)=\sum_{\substack{Y \in \mathcal{S} \mathcal{F}_{J} \\ f_{Y}=k}} \chi(Y) 2^{-\omega(Y)}
$$

for any integer $k \geq 0$. The sum $u(\chi, k)$ is the sum $a(\chi, \Psi,(1), J, k)$ defined by (3.18) where $\Psi$ is taken equal to the unit character. Let $\mathcal{C} \ell_{1}=\mathcal{C} \ell(R)_{1}$ denote the subgroup of $\mathcal{C} \ell(R)$ formed by the $\chi \in \mathcal{C} \ell(R)$ which are trivial on the subgroup $\operatorname{Ker} \varphi$. Then $\# \mathcal{C} \ell(R)_{1}=\#(\mathcal{C} \ell(R) / \operatorname{Ker} \varphi)=\#\left(\mathbb{Z} / \mathbb{Z} f_{0}\right)=f_{0}$. By (3.23),

$$
\left|\sum_{\substack{\chi \in \mathcal{C} \ell \\ \chi \notin \mathcal{C} \ell_{1}}} u(\chi, n)\right| \leq\left(h_{0}-f_{0}\right) \alpha_{1}(R) 2^{\omega(J)} n^{1 / 2} q^{n / 2} .
$$

Let $\chi \in \mathcal{C} \ell_{1}$ and let

$$
F(z)=\sum_{n=0}^{\infty} u(\chi, n)\left(\frac{z}{q}\right)^{n}
$$

By (3.21),

$$
\left(\frac{F(z)}{U(z)}\right)^{2}=L\left(\chi, \frac{z}{q}\right) G(z)
$$

with $U(z)$ and $G(z)$ given by (3.22) and (3.20). By (3.10), and then (2.8),

$$
L(\chi, z / q)=\frac{\left(1-(z / q)^{f_{0}}\right) P_{K}\left(\chi\left(I_{1}\right) z / q\right)}{\left(1-\chi\left(I_{1}\right) z / q\right)\left(1-\chi\left(I_{1}\right) z\right)}
$$

hence,

$$
F(z)=U(z) G(z)^{1 / 2} \frac{\left(1-(z / q)^{f_{0}}\right)^{1 / 2}\left(P_{K}\left(\chi\left(I_{1}\right) z / q\right)\right)^{1 / 2}}{\left(1-\chi\left(I_{1}\right) z / q\right)^{1 / 2}\left(1-\chi\left(I_{1}\right) z\right)^{1 / 2}} .
$$

Let $\eta \in] 0,1 / 2[$. According to [1, Lemma 2.1],

$$
\begin{aligned}
\mid \frac{u(\chi, n)}{q^{n}}-\chi\left(I_{1}\right)^{n} U & \left(\frac{1}{\chi\left(I_{1}\right)}\right) G\left(\frac{1}{\chi\left(I_{1}\right)}\right)^{1 / 2} \frac{\left(1-\left(\frac{1}{\chi\left(I_{1}\right) q}\right)^{f_{0}} P_{K}\left(\frac{1}{q}\right)\right)^{1 / 2}}{\pi^{1 / 2}(1-1 / q)^{1 / 2} n^{1 / 2}} \mid \\
& \leq \beta(q, \eta) \max \left\{|U(z)|\left|P_{K}(z / q) G(z)\right|^{1 / 2} ;|z|=q^{\eta}\right\} n^{-3 / 2}
\end{aligned}
$$

with $\beta(q, \eta)$ a constant. 
Since $n+f_{A} \equiv 0 \bmod f_{0}$ and $f_{I_{1}}=1$, we have $f_{A}+n f_{I_{1}} \equiv 0 \bmod f_{0}$. Since $\chi$ is trivial on $\operatorname{Ker} \varphi$, it follows that $\chi\left(I_{1}\right)^{n} \chi(A)=1$ and $\chi\left(I_{1}\right)^{f_{0}}=1$. By a proof which mimics that given in [1, Prop. 2.2], we get

$$
\begin{aligned}
& \mid \chi(A) u(\chi, n) q^{-n} \\
& \qquad \begin{aligned}
-U\left(\frac{1}{\chi\left(I_{1}\right)}\right) G\left(\frac{1}{\chi\left(I_{1}\right)}\right)^{1 / 2} P_{K}\left(\frac{1}{q}\right)^{1 / 2} & \frac{\left(1-(1 / q)^{f_{0}}\right)^{1 / 2}}{\pi^{1 / 2}(1-1 / q)^{1 / 2}} n^{-1 / 2} \mid \\
& \leq h \alpha_{3}(R, \eta) \lambda_{\eta}(J) n^{-3 / 2}
\end{aligned}
\end{aligned}
$$

with $\lambda_{\eta}$ defined by (3.29) and $\alpha_{3}(R, \eta)$ a constant.

Since $\chi$ is trivial on $\operatorname{Ker} \varphi$ and $f_{I_{1}}=1$, we have $\chi\left(I_{1}\right)^{f_{Z}}=\chi(Z)$ for any ideal $Z$ and by (3.22), (3.20) and (2.5),

$$
\begin{aligned}
& U\left(\frac{1}{\chi\left(I_{1}\right)}\right)=\prod_{\substack{P \in \mathcal{P} \\
P \mid A H}}\left(1+\frac{1}{2|P|}\right)^{-1}, \\
& G\left(\frac{1}{\chi\left(I_{1}\right)}\right)=\prod_{P \in \mathcal{P}}\left(1-\frac{3}{4|P|^{2}}-\frac{1}{4|P|^{3}}\right) .
\end{aligned}
$$

We conclude the proof by combining (1), (2), (4), (6), (7), (8), (2.4), (2.10) and (2.11), with $\alpha_{2}(R)=\left(h_{0}-f_{0}\right) \alpha_{1}(R)$.

Proposition 3.9. Let $H, A \in \mathcal{I}(R)$. Then, for any positive integer $n$ such that $n+f_{A} \equiv 0 \bmod f_{0}$,

$$
\begin{aligned}
\mid \sum_{\substack{Y \in \mathcal{S F}_{H} \\
f_{Y}=n \\
A Y \in \operatorname{Pr}}} \Omega(Y) 2^{-\omega(Y)}-B_{2}(R) & \Gamma(H) q^{n} n^{-1 / 2} \mid \\
& \leq \alpha_{4}(R) n q^{n / 2}+\alpha_{5}(R) q^{n} n^{-3 / 2}
\end{aligned}
$$

where

$$
\begin{aligned}
B_{2}(R) & =\left(\frac{h \varrho(R)}{\pi q^{g+f_{0}-1}}\right)^{1 / 2} \prod_{P \in \mathcal{P}}\left(1+\frac{1}{1+2|P|}\right)\left(1-\frac{1}{|P|}\right)^{1 / 2}, \\
\Gamma(H) & =\prod_{\substack{P \in \mathcal{P} \\
P \mid H}}\left(1+\frac{1}{1+2|P|}\right)^{-1}
\end{aligned}
$$

with $\alpha_{4}(R)$ and $\alpha_{5}(R)$ constants.

Proof. As for Proposition 3.8. We choose a particular value for $\eta$, for instance $\eta=1 / 4$. 
Proposition 3.10. Let $\eta \in] 0,1 / 2[$. Then, for $H, A \in \mathcal{I}(R)$ and any integer $n>0$,

$$
\sum_{\substack{Y \in \mathcal{S F}_{H} \\ f_{Y}=n \\ A Y \in \operatorname{Pr}}} \lambda_{\eta}(Y) 2^{-\omega(Y)} \leq \alpha_{6}(R, \eta) q^{n} n^{-1 / 2}
$$

with $\alpha_{6}(R, \eta)$ a constant.

Proof. Let

By orthogonality,

$$
x_{n}=\sum_{\substack{Y \in \mathcal{S F}_{H} \\ f_{Y}=n \\ A Y \in \operatorname{Pr}}} \lambda_{\eta}(Y) 2^{-\omega(Y)} .
$$

$$
h_{0} x_{n}=\sum_{\chi \in \widehat{\mathcal{C} \ell}} \chi(A) u(\chi, H, \eta, n)
$$

where

$$
u(\chi, H, \eta, n)=\sum_{\substack{Y \in \mathcal{S F}_{H} \\ f_{Y}=n}} \chi(Y) \lambda_{\eta}(Y) 2^{-\omega(Y)} .
$$

By (3.29), $\lambda_{\eta}(Y)>0$. Hence,

$$
|u(\chi, H, \eta, n)| \leq \sum_{\substack{Y \in \mathcal{S} \mathcal{F}_{H} \\ f_{Y}=n}} \lambda_{\eta}(Y) 2^{-\omega(Y)} .
$$

We consider the series

$$
f(z)=\sum_{n=0}^{\infty} u\left(\chi_{0}, H, \eta, n\right)\left(\frac{z}{q}\right)^{n}=\sum_{Y \in \mathcal{S F}_{H}} \lambda_{\eta}(Y) 2^{-\omega(Y)}\left(\frac{z}{q}\right)^{f_{Y}} .
$$

Proceeding as for Proposition 3.6 and Corollary 3.7, we get

$$
|u(\chi, H, \eta, n)| \leq \alpha_{6}(R, \eta) q^{n} n^{-1 / 2}
$$

with $\alpha_{6}(R, \eta)$ a constant.

4. Quadratic forms with coefficients in the ring $R=R_{\left\{v_{0}\right\}}$. Let $S$ be a non-empty finite set of places of $K$ containing $v_{0}$ and let $r=\# S$. For $v \in V(K)$ such that $v \neq v_{0}$, let $P_{v}$ denote the prime ideal of $R$ associated with the place $v$. For $r$-tuples $\mathbf{m}=\left(m_{v}\right)_{v \in S}$ and $\mathbf{n}=\left(n_{v}\right)_{v \in S}$ of rational integers, let $H(S, \mathbf{m}, \mathbf{n})$ denote the number of pairs $(a, b) \in R \times R$ such that (1) $\quad v(a)=m_{v}$ and $v(b)=n_{v}$ for all $v \in S$,

(2) the quadratic form

$$
X^{2}-a Y^{2}-b Z^{2}
$$

represents 0 over the field $K$. 
The goal of this section is to establish an estimate for $H(S, \mathbf{m}, \mathbf{n})$ with $-\sum_{v \in S} f_{v} m_{v}$ and $-\sum_{v \in S} f_{v} n_{v}$ positive. The proof will provide an estimate for the number $H_{1}(S, \mathbf{m}, \mathbf{n})$ of $(a, b) \in R \times R$ such that conditions (1) and (2) above are satisfied and

(3) the ideals $a\left(\prod_{v \in S-\left\{v_{0}\right\}} P_{v}^{-v(a)}\right)$ and $b\left(\prod_{v \in S-\left\{v_{0}\right\}} P_{v}^{-v(b)}\right)$ are squarefree and coprime,

and for the number $H^{\prime}(S, \mathbf{m}, \mathbf{n})$ of $(a, b) \in R^{2}$ such that (1) and (2) are satisfied and

$\left(3^{\prime}\right) \quad$ the ideals $a\left(\prod_{v \in S-\left\{v_{0}\right\}} P_{v}^{-v(a)}\right)$ and $b\left(\prod_{v \in S-\left\{v_{0}\right\}} P_{v}^{-v(b)}\right)$ are squarefree.

Let

$$
G=\prod_{\substack{v \in S \\ v \neq v_{0}}} P_{v}
$$

We note that $G=1$ when $S=\left\{v_{0}\right\}$.

For $a \in K$, let $\mathbf{v}(a)$ denote the $r$-tuple $(v(a))_{v \in S}$. For an $r$-tuple $\mathbf{m}=$ $\left(m_{v}\right)_{v \in S}$ of rational integers, let

$$
\|\mathbf{m}\|=-\sum_{v \in S} f_{v} m_{v}
$$

If $x \in R$, the principal ideal $R x$ may be written in a unique way as

$$
R x=\left(\prod_{\substack{v \in S \\ v \neq v_{0}}} P_{v}^{v(x)}\right) U(x)^{2} Q(x)
$$

with $U(x) \in \mathcal{I}(R)$ and $Q(x) \in \mathcal{S} \mathcal{F}(R)$ coprime to $G$.

Let $\mathcal{X}(S, \mathbf{m}, \mathbf{n})$ denote the set of $(a, b) \in R \times R$ such that $v(a)=m_{v}$ and $v(b)=n_{v}$ for all $v \in S$. If $(a, b) \in \mathcal{X}(S, \mathbf{m}, \mathbf{n})$, let $D(a, b)=$ g.c.d. $(Q(a), Q(b))$ and let $J_{a, b}(a)$ and $J_{a, b}(b)$ be the square-free ideals defined by $Q(a)=$ $D(a, b) J_{a, b}(a)$ and $Q(b)=D(a, b) J_{a, b}(b)$. Then $J_{a, b}(a)$ and $J_{a, b}(b)$ are coprime and

$$
\begin{aligned}
f_{J_{a, b}(a)}+2 f_{U(a)}+f_{D(a, b)} & =\|\mathbf{m}\|, \\
f_{J_{a, b}(b)}+2 f_{U(b)}+f_{D(a, b)} & =\|\mathbf{n}\| .
\end{aligned}
$$

For a square-free ideal $D$ of $R$ coprime to $G$, and for ideals $U$ and $V$ of $R$ coprime to $G$ such that $2 f_{U}+f_{D} \leq\|\mathbf{m}\|$ and $2 f_{V}+f_{D} \leq\|\mathbf{n}\|$, let $\mathcal{Y}(S, \mathbf{m}, \mathbf{n}, D, U, V)$ denote the set of $(a, b) \in \mathcal{X}(S, \mathbf{m}, \mathbf{n})$ such that $U(a)=U$, $U(b)=V$, and $D(a, b)=D$, and let $\mathcal{Z}(S, \mathbf{m}, \mathbf{n}, D, U, V)$ denote the set of $(a, b) \in \mathcal{Y}(S, \mathbf{m}, \mathbf{n}, D, U, V)$ such that the quadratic form $\left(f_{a, b}\right)$ represents 0 
over $K$. Let $Z(S, \mathbf{m}, \mathbf{n}, D, U, V)=\# \mathcal{Z}(S, \mathbf{m}, \mathbf{n}, D, U, V)$. Then

$$
H(S, \mathbf{m}, \mathbf{n})=\sum_{\substack{D \in \mathcal{S} \mathcal{F}_{G} \\ f_{D} \leq \min (\|\mathbf{m}\|,\|\mathbf{n}\|)}} \sum_{\substack{U \in \mathcal{I}_{G} \\ 2 f_{U}+f_{D} \leq\|\mathbf{m}\|}} \sum_{\substack{V \in \mathcal{I}_{G} \\ 2 f_{V}+f_{D} \leq\|\mathbf{n}\|}} Z(S, \mathbf{m}, \mathbf{n}, D, U, V) .
$$

We fix $\mathbf{m}$ and $\mathbf{n}$ with $\|\mathbf{m}\|$ and $\|\mathbf{n}\|$ positive. By symmetry, we can and will suppose that

$$
\|\mathbf{m}\| \leq\|\mathbf{n}\| .
$$

We fix a square-free ideal $D$ in $R$, and ideals $U$ and $V$ in $R$ such that $f_{D}+2 f_{U} \leq\|\mathbf{m}\|$ and $f_{D}+2 f_{V} \leq\|\mathbf{n}\|$, all coprime to $G$. For brevity, set

$$
\begin{aligned}
\mathfrak{A} & =D U^{2}\left(\prod_{v \in S-\left\{v_{0}\right\}} P_{v}^{m_{v}}\right), & \mathfrak{B} & =D V^{2}\left(\prod_{v \in S-\left\{v_{0}\right\}} P_{v}^{n_{v}}\right), \\
\mathcal{Y} & =\mathcal{Y}(S, \mathbf{m}, \mathbf{n}, D, U, V), & \mathcal{Z} & =\mathcal{Z}(S, \mathbf{m}, \mathbf{n}, D, U, V), \\
M & =\|\mathbf{m}\|-2 f_{U}-f_{D}, & N & =\|\mathbf{n}\|-2 f_{V}-f_{D},
\end{aligned}
$$

and for $(a, b) \in \mathcal{Y}(S, \mathbf{m}, \mathbf{n}, D, U, V)$, write $J(a)=J_{(a, b)}(a)$ and $J(b)=$ $J_{(a, b)}(b)$. In view of (4.4),

$$
(a, b) \in \mathcal{Y}(S, \mathbf{m}, \mathbf{n}, D, U, V) \Rightarrow f_{J(a)}=M, f_{J(b)}=N .
$$

Now, we suppose that

$$
M+f_{D} \leq \frac{\log q}{\log 2} N .
$$

Let $\mathcal{A}$, resp. $\mathcal{B}$, denote the first, resp. second projection of the set $\mathcal{Y}=$ $\mathcal{Y}(\mathbf{m}, \mathbf{n}, D, U, V)$. For $a \in \mathcal{A}$, let ${ }_{a} \mathcal{Y}$ denote the set of $b \in R$ such that $(a, b) \in \mathcal{Y}$. Similarly, for $b \in \mathcal{B}$, let $\mathcal{Y}_{b}$ denote the set of $a \in R$ such that $(a, b) \in \mathcal{Y}$.

Our proof makes use of characters of order 2 of the multiplicative group $K^{\star}$, defined as follows. For any $v \in V(K)$ we have chosen a uniformizing element $\pi_{v} \in K$. With this choice, every non-zero $\alpha \in K$ is uniquely written as a product

$$
\alpha=\operatorname{sgn}_{v}(\alpha) \pi_{v}^{v(\alpha)} u_{v}(\alpha)
$$

with $\operatorname{sgn}_{v}(\alpha) \in k_{v}$ and $u_{v}(\alpha) \in U_{v}^{(1)}$. Let

$$
\theta_{v}(\alpha)= \begin{cases}1 & \text { if } \operatorname{sgn}_{v}(\alpha) \text { is a square in } k_{v}, \\ -1 & \text { otherwise. }\end{cases}
$$

The character $\Theta_{\emptyset}$ is the unit character. If $\Sigma$ is a non-empty finite set of places of $K$, the character $\Theta_{\Sigma}$ is defined by

$$
\Theta_{\Sigma}(a)=\prod_{v \in \Sigma} \theta_{v}(a)
$$


Let $(a, b)$ be a pair of non-zero elements of $K$. For $v \in V=V(K)$, the Hilbert symbol $(a, b)_{v}$ is defined by

$$
(a, b)_{v}= \begin{cases}1 & \text { if }\left(f_{(a, b)}\right) \text { represents } 0 \text { over } K_{v}, \\ -1 & \text { if not. }\end{cases}
$$

It is well known that

$$
(a, b)_{v}=\theta_{v}(-1)^{v(a) v(b)} \theta_{v}\left(\operatorname{sgn}_{v}(a)\right)^{v(b)} \theta_{v}\left(\operatorname{sgn}_{v}(b)\right)^{v(a)}
$$

(cf. [7, Chap. XIV, 4]), and that the Hilbert symbol satisfies the product formula

$$
\prod_{v \in V(K)}(a, b)_{v}=1
$$

(cf. [7, Chap. XIV, annexe]). If $\Sigma$ is a finite set of places of $K$, let

$$
(a, b)_{\Sigma}=\prod_{v \in \Sigma}(a, b)_{v},
$$

the empty product being equal to 1 .

If $H \in \mathcal{I}(R)$, let $\Sigma(H)=\left\{v_{P} ; P \in \mathcal{P}(R), P \mid H\right\}$. Let $T$ denote the set of $v \in S$ such that $m_{v}$ or $n_{v}$ is odd, and let $\tau=\tau(\mathbf{m}, \mathbf{n})=\# T$.

Remark 4.1. For $(a, b) \in \mathcal{Y}(\mathbf{m}, \mathbf{n}, D, U, V)$, let

$$
W(a, b)=T \cup\left\{v_{P} ; P \mid D J(a) J(b)\right\} .
$$

Then

$$
\begin{gathered}
(a, b)_{v}=1 \quad \text { for } v \notin W(a, b), \\
(a, b)_{W(a, b)}=1 .
\end{gathered}
$$

Proof. Let $(a, b) \in \mathcal{Y}(\mathbf{m}, \mathbf{n}, D, U, V)$. If $P \in \mathcal{P}(R)$ does not divide $D J_{(a, b)}(a) J_{(a, b)}(b)$, then $v_{P}(a) \equiv 0 \bmod 2, v_{P}(b) \equiv 0 \bmod 2$, and by (4.15), $(a, b)_{v_{P}}=1$. Similarly, if $v \in S$ is such that $m_{v}$ and $n_{v}$ are even, then $(a, b)_{v}=1$. Hence, $(a, b)_{v}=1$ for any $v \notin W(a, b)$.

By the product formula (4.16),

$$
1=\prod_{v \in V(K)}(a, b)_{v}=\prod_{\substack{v \in V(K) \\ v \notin W(a, b)}}(a, b)_{v} \cdot \prod_{v \in W(a, b)}(a, b)_{v}=(a, b)_{W(a, b)}
$$

with notation (4.17).

Proposition 4.2. We have

$$
\begin{aligned}
2^{\tau+\omega(D)} Z & =\sum_{(a, b) \in \mathcal{Y}} 2^{-\omega(J(a) J(b))} \sum_{X \subset W(a, b)}(a, b)_{X}, \\
2^{\omega(D)} Z & =Z_{1}+Z_{2}+Z_{3},
\end{aligned}
$$


with

$$
\begin{aligned}
& Z_{1}=2 \sum_{t \subset T} \sum_{(a, b) \in \mathcal{Y}} 2^{-\omega(J(a) J(b))}(a, b)_{t}, \\
& Z_{2}=2 \sum_{t \subset T} \sum_{(a, b) \in \mathcal{Y}} 2^{-\omega(J(a) J(b))}(a, b)_{t} \sum_{\substack{B \in \mathcal{I} \\
B \mid J(b) \\
B \neq(1)}}(a, b)_{\Sigma(B)}, \\
& Z_{3}=\sum_{t \subset T} \sum_{(a, b) \in \mathcal{Y}} 2^{-\omega(J(a) J(b))}(a, b)_{t} \sum_{\substack{H \in \mathcal{I} \\
H \mid D J(a)}} \sum_{\substack{B \in \mathcal{I} \\
B \mid J(b)}}(a, b)_{\Sigma(H B)} .
\end{aligned}
$$

Proof. Let $(a, b) \in \mathcal{Y}(\mathbf{m}, \mathbf{n}, D, U, V)$. By the Hasse principle, $\left(f_{a, b}\right)$ represents 0 over $K$ if and only if it represents 0 over any $K_{v}$ with $v$ running through $V(K)$. In view of (4.14) and (4.18), $(a, b) \in \mathcal{Z}=\mathcal{Z}(\mathbf{m}, \mathbf{n}, D, U, V)$ if and only if

$$
\prod_{v \in W(a, b)}\left(1+(a, b)_{v}\right)=2^{\# W(a, b)} .
$$

Otherwise this product is 0 . In view of the definition of the set $W(a, b)$,

$$
2^{\# W(a, b)}=2^{\tau+\omega(D)+\omega(J(a) J(b))},
$$

thus,

$$
2^{\tau+\omega(D)} Z=\sum_{(a, b) \in \mathcal{Y}} 2^{-\omega(J(a) J(b))} \prod_{v \in W(a, b)}\left(1+(a, b)_{v}\right) .
$$

Expanding this product and using notation (4.17), we get

$$
2^{\tau+\omega(D)} Z=\sum_{(a, b) \in \mathcal{Y}} 2^{-\omega(J(a) J(b))} \sum_{X \subset W(a, b)}(a, b)_{X} .
$$

As in the proof of Proposition 3.3 in [1], following Hooley's idea, we split the right hand side of (1) into three subsums $Z_{i}, 1 \leq i \leq 3$, corresponding to different subsets $X \subset W(a, b)$.

1) The sum $Z_{1}$ which will give the main term contains for each $(a, b) \in \mathcal{Y}$ all subsets $t$ and $t \cup \Sigma(D J(a) J(b))$ with $t \subset T$, that is,

$$
Z_{1}=\sum_{t \subset T} \sum_{(a, b) \in \mathcal{Y}} 2^{-\omega(J(a) J(b))}\left((a, b)_{t}+(a, b)_{t \cup \Sigma(D J(a) J(b))}\right) .
$$

For any $t \subset T$, let $t^{\prime}$ denote the difference set $T-t$. The map $t \mapsto t^{\prime}$ being a permutation of the subsets of $T$,

$$
Z_{1}=\sum_{t \subset T} \sum_{(a, b) \in \mathcal{Y}} 2^{-\omega(J(a) J(b))}\left((a, b)_{t}+(a, b)_{t^{\prime} \cup \Sigma(D J(a) J(b))}\right) .
$$

For $(a, b) \in \mathcal{Y}$ and $t \subset T$,

$$
(a, b)_{t^{\prime} \cup \Sigma(D J(a) J(b))}=\left((a, b)_{t}\right)^{2}(a, b)_{t^{\prime} \cup \Sigma(D J(a) J(b))} .
$$


By (4.17),

$$
(a, b)_{t^{\prime} \cup \Sigma(D J(a) J(b))}=(a, b)_{t}(a, b)_{T \cup \Sigma(D J(a) J(b))}=(a, b)_{t}(a, b)_{W(a, b)},
$$

and by (4.19),

$$
Z_{1}=2 \sum_{t \subset T} \sum_{(a, b) \in \mathcal{Y}} 2^{-\omega(J(a) J(b))}(a, b)_{t} .
$$

2) The sum $Z_{2}$ contains for each $(a, b) \in \mathcal{Y}$ all subsets $t \cup \Sigma(B)$ and $t \cup \Sigma\left(D J(a) B^{\prime}\right)$ with $t \subset T, B \neq(1)$ running over the ideals dividing $J(b)$, and $B^{\prime} \neq J(b)$ running over the ideals dividing $J(b)$, that is,

$$
\begin{gathered}
Z_{2}=\sum_{t \subset T} \sum_{(a, b) \in \mathcal{Y}} 2^{-\omega(J(a) J(b))} \sum_{\substack{B \in \mathcal{I} \\
B \mid J(b) \\
B \neq(1)}}(a, b)_{t \cup \Sigma(B)} \\
+\sum_{t^{\prime} \subset T} \sum_{\substack{B^{\prime} \in \mathcal{I} \\
B^{\prime} \mid J(b) \\
B^{\prime} \neq J(b)}}(a, b)_{t^{\prime} \cup \Sigma\left(D J(a) B^{\prime}\right)},
\end{gathered}
$$

where $t^{\prime}$ has the same meaning as above. If $B^{\prime} \in \mathcal{I}$ divides $J(b)$, then $J(b)=$ $B B^{\prime}$. Moreover, $B^{\prime} \neq J(b)$ if and only if $B \neq(1)$. With notation (4.17) we find that for any subset $t \subset T$,

$$
(a, b)_{t^{\prime} \cup \Sigma\left(B^{\prime}\right)}=(a, b)_{t^{\prime} \cup \Sigma\left(B^{\prime}\right)}\left((a, b)_{t \cup \Sigma(B)}\right)^{2}=(a, b)_{T \cup \Sigma(J(b))}(a, b)_{t \cup \Sigma(B)},
$$

whence

$$
Z_{2}=\sum_{t \subset T} \sum_{(a, b) \in \mathcal{Y}} 2^{-\omega(J(a) J(b))}(a, b)_{t} \sum_{\substack{B \in \mathcal{I} \\ B \mid J(b) \\ B \neq(1)}}(a, b)_{\Sigma(B)}\left(1+(a, b)_{W(a, b)}\right) .
$$

We now get (4.23) from (4.19).

3) The sum $Z_{3}$ contains the remaining terms, which yields (4.24).

We compute $Z_{1}$ and we bound $Z_{2}$ and $Z_{3}$. Once more, we need new notations. Let $T_{1,0}, T_{0,1}$, and $T_{1,1}$ denote respectively the sets of $v \in S$ such that $m_{v}$ is odd and $n_{v}$ is even; $m_{v}$ is even and $n_{v}$ is odd; $m_{v}$ and $n_{v}$ are odd. We denote by $J(\mathcal{A})$ and $J(\mathcal{B})$ respectively the sets of ideals $J(a)$ with $a$ running over $\mathcal{A}$, and the set of ideals $J(b)$ with $b$ running over $\mathcal{B}$.

Proposition 4.3. We have

$$
\begin{aligned}
\left|Z_{1}-2 L\right| & \leq \beta_{1}(R) 2^{\tau+r+f_{G} / 2} 2^{\omega(D)+N / 2}\left(N^{1 / 2} q^{M+N / 2}+M^{1 / 2} q^{N+M / 2}\right) \\
\left|Z_{2}\right| & \leq \beta_{1}(R) 2^{\tau+r+f_{G} / 2} 2^{\omega(D)+N / 2} M^{1 / 2} q^{N+M / 2}
\end{aligned}
$$


where

$$
L=L(S, \mathbf{m}, \mathbf{n}, D, U, V)=\sum_{(a, b) \in \mathcal{Y}} 2^{-\omega(J(a) J(b))},
$$

with $\beta_{1}(R)$ and $\beta_{2}(R)$ constants.

Proof. For $i=1,2$, let

$$
S_{i}=\sum_{t \subset T} \sum_{(a, b) \in \mathcal{Y}} 2^{-\omega(J(a) J(b))}(a, b)_{t} \sigma_{i}(a, b)
$$

with

$$
\begin{aligned}
& \sigma_{1}(a, b)=1 \\
& \sigma_{2}(a, b)=\sum_{\substack{B \in \mathcal{I} \\
B \mid J(b) \\
B \neq(1)}}(a, b)_{\Sigma(B)} .
\end{aligned}
$$

Let $(a, b) \in \mathcal{Y}(S, \mathbf{m}, \mathbf{n}, D, U, V)$. By (4.15), for $v \in S$,

$$
(a, b)_{v}=\theta_{v}(-1)^{m_{v} n_{v}} \theta_{v}\left(\operatorname{sgn}_{v}(b)\right)^{m_{v}} \theta_{v}\left(\operatorname{sgn}_{v}(a)\right)^{n_{v}}
$$

with $\theta_{v}$ defined by (4.12). With notation (4.13),

$$
S_{i}=\sum_{\substack{t_{1,0} \subset T_{1,0} \\ t_{0,1} \subset T_{0,1} \\ t_{1,1} \subset T_{1,1}}} \sum_{(a, b) \in \mathcal{Y}} 2^{-\omega(J(a) J(b))} \Theta_{t_{1,0}}(b) \Theta_{t_{0,1}}(a) \Theta_{t_{1,1}}(-a b) \sigma_{i}(a, b) .
$$

We look at $S_{2}$. Let $(a, b) \in \mathcal{Y}$. In view of (4.3), if $P \in \mathcal{P}(R)$ divides $J(b)$, then $v_{P}(b) \equiv 1 \bmod 2, v_{P}(a) \equiv 0 \bmod 2$, and by $(4.15),(a, b)_{v_{P}}=$ $\theta_{v_{P}}\left(\operatorname{sgn}_{P}(a)\right)$. Hence, with notations (4.13) and (4.17), if $B \in \mathcal{I}(R)$ divides $J(b)$, then $(a, b)_{\Sigma(B)}=\Theta_{\Sigma(B)}(a)$. Let $b \in \mathcal{B}$. Every $x \in \mathcal{Y}_{b}$ may be written as a product $\alpha a$ with $\alpha \in k^{\star}$ and $a \in \mathcal{M}$, where $\mathcal{M}$ is the set of monic elements. Moreover, $a \in \mathcal{M} \cap \mathcal{Y}_{b}$. Hence, by (4) and (3),

$$
\begin{aligned}
S_{2}= & \left\{\sum_{b \in \mathcal{B}} 2^{-\omega(J(b))} \sum_{a \in \mathcal{M} \cap \mathcal{Y}_{b}} 2^{-\omega(J(a))}\right\} \\
& \times\left\{\sum_{\substack{t_{1,0} \subset T_{1,0} \\
t_{0,1} \subset T_{0,1} \\
t_{1,1} \subset T_{1,1}}} \Theta_{t_{1,0}}(b) \Theta_{t_{0,1}}(a) \Theta_{t_{1,1}}(-a b) \sum_{\substack{B \in \mathcal{I} \\
B \mid J(b) \\
B \neq(1)}} \Theta_{\Sigma(B)}(a)\right. \\
& \left.\times \sum_{\alpha \in k^{\star}} \Theta_{t_{0,1}}(\alpha) \Theta_{t_{1,1}}(\alpha) \Theta_{\Sigma(B)}(\alpha)\right\} .
\end{aligned}
$$

We consider the inner sum

$$
\Omega_{t_{0,1}, t_{1,1}, B}=\sum_{\alpha \in k^{\star}} \Theta_{t_{0,1} \cup t_{1,1} \cup \Sigma(B)}(\alpha) .
$$


Let $v \in t_{0,1} \cup t_{1,1} \cup\left\{v_{P} ; P \mid B\right\}$. If $f_{v}$ is even, then every $\alpha \in k^{\star}$ is a square in the field $k_{v}$ and by (4.12), $\theta_{v}(\alpha)=1$. If $f_{v}$ is odd, then by (4.12), $\theta_{v}(\alpha)=1$ or -1 according as $\alpha$ is or is not a square in $k$. Hence, $\Omega_{t_{0,1}, t_{1,1}, B}=q-1$ or $\Omega_{t_{0,1}, t_{1,1}, B}=0$ according as the sum

$$
\sum_{v \in t_{0,1}} f_{v}+\sum_{v \in t_{1,1}} f_{v}+\sum_{\substack{P \in \mathcal{P} \\ P \mid B}} f_{v_{P}}
$$

is even or odd. With the same arguments, looking at the inner sum

$$
\sum_{\beta \in k^{\star}} \Theta_{t_{1,0}}(\beta) \Theta_{t_{1,1}}(\beta)
$$

we conclude that in the sum $S_{2}$, there only occur the 4 -tuples $\left(t_{0,1}, t_{1,0}, t_{1,1}, B\right)$ such that the sums

$$
\sum_{v \in t_{0,1}} f_{v}+\sum_{v \in t_{1,1}} f_{v}+\sum_{\substack{P \in \mathcal{P} \\ P \mid B}} f_{v_{P}}, \quad \sum_{v \in t_{1,0}} f_{v}+\sum_{v \in t_{1,1}} f_{v}
$$

are even. In the following, we denote this condition and analogous parity conditions by the symbol $\left(t_{0,1}, t_{1,0}, t_{1,1}, B\right) \equiv 0$. Hence,

$$
\begin{aligned}
S_{2}= & \sum_{b \in \mathcal{B}} 2^{-\omega(J(b))} \sum_{\substack{t_{1,0} \subset T_{1,0} \\
t_{0,1} \subset T_{0,1} \\
t_{1,1} \subset T_{1,1}}} \sum_{\begin{array}{c}
B \in \mathcal{I} \\
B \mid J(b) \\
B \neq(1) \\
\left(t_{0,1}, t_{1,0}, t_{1,1}, B\right) \equiv 0
\end{array}} \Theta_{t_{1,0}}(b) \Theta_{t_{1,1}}(-b) \\
& \times \sum_{a \in \mathcal{Y}_{b}} 2^{-\omega(J(a))} \Theta_{t_{0,1} \cup t_{1,1} \cup \Sigma(B)}(a) .
\end{aligned}
$$

We consider the last inner sum. By the parity condition, the map

$$
y \mapsto \Theta_{t_{0,1} \cup t_{1,1} \cup \Sigma(B)}(y)
$$

is trivial on the group $k^{\star}$. Hence, we may define a morphism $\Psi^{\prime}$ from $\operatorname{Pr}(R)$, the monoid of non-zero principal ideals of $R$, to the group $\{1,-1\}$ by $\Psi^{\prime}(Y)=\Theta_{t_{0,1} \cup t_{1,1} \cup \Sigma(B)}(\eta)$ if $\eta \in R$ generates the principal ideal $Y$. This morphism extends in an obvious unique way to a morphism from the group $\operatorname{FPr}(R)$ of principal fractional ideals to $\{1,-1\}$. Since $\operatorname{FPr}(R)$ has finite index in the group $\mathcal{F}(R)$, the morphism $\Psi^{\prime}$ extends to a morphism $\Psi$ from $\mathcal{F}(R)$ to the group $\mu_{l}$ of $l$ th roots of 1 for some $l$. In view of the definition of the set $\mathcal{Y}_{b}$,

$$
\sum_{a \in \mathcal{Y}_{b}} 2^{-\omega(J(a))} \Theta_{t_{0,1} \cup t_{1,1} \cup \Sigma(B)}(a)=(q-1) \sum_{\substack{A \in \mathcal{S F}_{G D J(b)} \\ A \mathfrak{A} \in \operatorname{Pr} \\ f_{A}=M}} 2^{-\omega(A)} \Psi(\mathfrak{A} A)
$$

with $\mathfrak{A}$ defined by (4.7). Since $B \neq(1)$, the map

$$
y \mapsto \Theta_{t_{0,1} \cup t_{1,1} \cup \Sigma(B)}(y)
$$


is not trivial on $R$, and the morphism $\Psi$ is not trivial on the group of principal ideals. Moreover, $\Psi$ satisfies condition (3.16) with $H=B G$. Hence, by $(3.25)$,

$$
\begin{aligned}
& \left|\sum_{a \in \mathcal{Y}_{b}} 2^{-\omega(J(a))} \Theta_{t_{0,1} \cup t_{1,1} \cup \Sigma(B)}(a)\right| \\
& \quad \leq(q-1) \alpha_{1}(R) 2^{r+f_{G} / 2+f_{B} / 2} 2^{\omega(D J(b))} M^{1 / 2} q^{M / 2} .
\end{aligned}
$$

By (5) and (6),

$$
\left|S_{2}\right| \leq(q-1) \alpha_{1}(R) 2^{r+f_{G} / 2} 2^{\omega(D)} M^{1 / 2} q^{M / 2} \sum_{b \in \mathcal{B}} \sum_{\begin{array}{c}
t_{1,0} \subset T_{1,0} \\
t_{0,1} \subset T_{0,1} \\
t_{1,1} \subset T_{1,1}
\end{array}} \sum_{\substack{B \in \mathcal{I} \\
B \mid J(b) \\
B \neq(1)}} 2^{f_{B} / 2} .
$$

In view of (4.7) and the definition of $\mathcal{B}$, if $B \in \mathcal{I}(R)$ divides $J(b)$ with $b \in \mathcal{B}$, then $B$ is square-free and coprime to $G D, R b=\mathfrak{B} B B^{\prime}$ with $B^{\prime}$ square-free and coprime to $G D B$. Hence,

$$
\left|S_{2}\right| \leq(q-1)^{2} \alpha_{1}(R) 2^{\tau+r+f_{G} / 2} 2^{\omega(D)} M^{1 / 2} q^{M / 2} \mathcal{S}
$$

with

$$
\mathcal{S}=\sum_{\substack{B \in \mathcal{S F}_{G D} \\ 1 \leq f_{B} \leq N}} 2^{f_{B} / 2} \sum_{\substack{B^{\prime} \in \mathcal{S} \mathcal{F}_{G D B} \\ \mathfrak{B} B B^{\prime} \in \operatorname{Pr} \\ f_{B B^{\prime}}=N}} 1
$$

The ideals $B^{\prime}$ occurring in the inner sum above belong to the same ideal class. Hence, by (3.5),

$$
\mathcal{S} \leq \varrho(R) \sum_{\substack{B \in \mathcal{I}_{G D} \\ 1 \leq f_{B} \leq N}} 2^{f_{B} / 2} q^{N-f_{B}} \leq \varrho(R) q^{N} \sum_{\substack{B \in \mathcal{I} \\ 1 \leq f_{B} \leq N}} 2^{f_{B} / 2} q^{-f_{B}} .
$$

Then, by (3.5),

$$
\mathcal{S} \leq h \frac{\sqrt{2}}{\sqrt{2}-1} \varrho(R)^{2} 2^{N / 2} q^{N} .
$$

This together with (7) gives (4.26).

Now, we deal with $S_{1}$. We break the sum (4) into three parts. The first part which will give the main term is given by the triple $\left(t_{0,1}, t_{1,0}, t_{1,1}\right)=$ $(\emptyset, \emptyset, \emptyset)$; the second part is given by the triples $\left(t_{0,1}, t_{1,0}, t_{1,1}\right)=\left(\emptyset, t_{1,0}, \emptyset\right)$ with $t_{1,0} \neq \emptyset$; and the third part contains the remaining terms. In other words,

$$
S_{1}=S_{1,1}+S_{1,2}+S_{1,3}
$$

with

$$
S_{1,1}=\sum_{(a, b) \in \mathcal{Y}} 2^{-\omega(J(a) J(b))},
$$




$$
S_{1,2}=\sum_{(a, b) \in \mathcal{Y}} 2^{-\omega(J(a) J(b))} \sum_{\substack{t_{1,0} \subset T_{1,0} \\ t_{1,0} \neq \emptyset}} \Theta_{t_{1,0}}(b),
$$

$$
S_{1,3}=\sum_{(a, b) \in \mathcal{Y}} 2^{-\omega(J(a) J(b))} \sum_{\substack{\left.t_{1,0} \subset T_{1,0} \\ t_{0,1} \subset T_{0,1} \\ t_{1,1} \subset T_{1,1} \\ t_{0,1}, t_{1,1}\right) \neq(\emptyset, \emptyset)}} \Theta_{t_{1,0}}(b) \Theta_{t_{0,1}}(a) \Theta_{t_{1,1}}(-a b) .
$$

We deal with $S_{1,2}$ and $S_{1,3}$ just as we have dealt with $S_{2}$. We get

$$
\begin{aligned}
& S_{1,2} \leq(q-1) \alpha_{1}(R) 2^{r+f_{G} / 2} \lambda_{1,2} 2^{\omega(D)} N^{1 / 2} q^{N / 2} \# \mathcal{A}, \\
& S_{1,3} \leq(q-1) \alpha_{1}(R) 2^{r+f_{G} / 2} \lambda_{1,3} 2^{\omega(D)} M^{1 / 2} q^{M / 2} \# \mathcal{B},
\end{aligned}
$$

where $\lambda_{1,2}$ is the number of $t_{1,0} \neq \emptyset$ such that $\left(\emptyset, t_{1,0}, \emptyset\right) \equiv 0$, and $\lambda_{1,3}$ is the number of $\left(t_{0,1}, t_{1,1}\right)$ with $\left(t_{0,1}, t_{1,1}\right) \neq(\emptyset, \emptyset)$ such that $\left(t_{0,1}, \emptyset, t_{1,1}\right) \equiv 0$. By $(3.5)$,

$$
\# \mathcal{A} \leq(q-1) \varrho(R) q^{M}, \quad \# \mathcal{B} \leq(q-1) \varrho(R) q^{N} .
$$

Hence,

$$
\left|S_{1,2}+S_{1,3}\right| \leq(q-1)^{2} \varrho(R) \alpha_{1}(R) 2^{\tau+r+f_{G} / 2}\left(N^{1 / 2} q^{M+N / 2}+M^{1 / 2} q^{N+M / 2}\right),
$$

since $\lambda_{1,2}+\lambda_{1,3} \leq \tau$. This together with (10) and (11) gives (4.25).

Proposition 4.4. Let $\theta \in] \log 2 / \log q, 1]$. Suppose that $\theta N \leq M \leq N$. Then

$$
\left|L-(q-1)^{2} B_{3}(R) \Lambda(G D) \frac{q^{M+N}}{\sqrt{M N}}\right| \leq \beta_{3}(R, \theta) 2^{r+\omega(D)} \frac{q^{M+N}}{M N}
$$

with

$$
\begin{aligned}
B_{3}(R) & =\frac{h \varrho(R)}{\pi q^{g+f_{0}-1}} \prod_{P \in \mathcal{P}}\left(1-\frac{1}{|P|^{2}}\right), \\
\Lambda(H) & =\prod_{\substack{P \in \mathcal{P} \\
P \mid H}}\left(1+\frac{1}{|P|}\right)^{-1}
\end{aligned}
$$

for any ideal $H$, and $\beta_{3}(R, \theta)$ a constant.

Proof. In view of (4.27) and the definition of the sets $\mathcal{Y}, \mathcal{A}$ and ${ }_{a} \mathcal{Y}$,

$$
L=\sum_{a \in \mathcal{A}} 2^{-\omega(J(a))} \eta(a)
$$

with

$$
\eta(a)=\sum_{b \in_{a} \mathcal{Y}} 2^{-\omega(J(b))} .
$$


In view of (4.7) and the definition of the sets ${ }_{a} \mathcal{Y}$,

$$
\eta(a)=(q-1) \sum_{\substack{B \in \mathcal{S} \mathcal{F}_{G D J(a)} \\ f_{B}=N \\ B \mathfrak{B} \in \operatorname{Pr}}} 2^{-\omega(B)} .
$$

Proposition 3.8 gives

(3) $\quad\left|\eta(a)-(q-1) B_{1}(R) \Omega(G D J(a)) q^{N} N^{-1 / 2}\right|$

$$
\leq(q-1)\left(\alpha_{2}(R) 2^{\omega(G D J(a))} N^{1 / 2} q^{N / 2}+\alpha_{3}(R, 1 / 4) \lambda_{1 / 4}(G D J(a)) q^{N} N^{-3 / 2}\right)
$$

with $B_{1}(R)$ defined by (3.27) and $\Omega(H)$ defined by (3.28). In view of (4.7) and the definition of the set $\mathcal{A}$,

$$
\sum_{a \in \mathcal{A}} 2^{-\omega(J(a))} \Omega(J(a))=(q-1) \sum_{\substack{A \in \mathcal{S} \mathcal{F}_{G D} \\ \mathfrak{A} A \in \mathrm{Pr} \\ f_{A}=M}} 2^{-\omega(A)} \Omega(A),
$$

and by (1) and (3),

$$
\begin{aligned}
\mid L-(q-1)^{2} B_{1}(R) \Omega( & G D) q^{N} N^{-1 / 2} S \mid \\
\leq & (q-1) \alpha_{2}(R) 2^{r+\omega(D)} N^{1 / 2} q^{N / 2} \# \mathcal{A} \\
& \quad+(q-1)^{2} \alpha_{3}(R, 1 / 4) \lambda_{1 / 4}(G D) q^{N} N^{-3 / 2} S^{\prime}
\end{aligned}
$$

with

$$
\begin{aligned}
S & =\sum_{\substack{A \in \mathcal{S F}_{G D} \\
f_{A}=M \\
A \mathfrak{A} \in \operatorname{Pr}}} 2^{-\omega(A)} \Omega(A), \\
S^{\prime} & =\sum_{\substack{A \in \mathcal{S F}_{G D} \\
f_{A}=M \\
A \mathfrak{A} \in \operatorname{Pr}}} 2^{-\omega(A)} \lambda_{1 / 4}(A) .
\end{aligned}
$$

By (3.30) and (3.33),

$$
\begin{gathered}
\left|S-B_{2}(R) \Gamma(G D) q^{M} M^{-1 / 2}\right| \leq \alpha_{4}(R) M q^{M / 2}+\alpha_{5}(R) q^{M} M^{-3 / 2}, \\
S^{\prime} \leq \alpha_{6}(R, 1 / 4) q^{M} M^{-1 / 2},
\end{gathered}
$$

with $B_{2}(R)$ and $\Gamma(H)$ defined by (3.31) and (3.32). We have seen above that $\# \mathcal{A} \leq(q-1) \varrho(R) q^{M}$. Hence, by (4), (7), and (8),

$$
\begin{aligned}
&\left|L-(q-1)^{2} B_{1}(R) B_{2}(R) \Omega(G D) \Gamma(G D) \frac{q^{M+N}}{\sqrt{M N}}\right| \\
& \leq B_{1}(R) \Theta(G D)(q-1)^{2}\left(\alpha_{4}(R) M q^{M / 2}+\alpha_{5}(R) q^{M} M^{-3 / 2}\right) \\
&+(q-1)^{2} \alpha_{2}(R) \varrho(R) 2^{r+\omega(D)} N^{1 / 2} q^{M+N / 2} \\
&+(q-1)^{2} \alpha_{3}(R, 1 / 4) \alpha_{6}(R, 1 / 4) \lambda_{1 / 4}(G D) q^{M+N} M^{-1 / 2} N^{-3 / 2} .
\end{aligned}
$$


By (3.27) and (3.31),

$$
B_{1}(R) B_{2}(R)=\frac{h \varrho(R)}{\pi q^{g+f_{0}-1}} \prod_{P \in \mathcal{P}}\left(1-\frac{1}{|P|}\right)\left(1+\frac{1}{|P|}\right) .
$$

By (3.28) and (3.32),

$$
\Gamma(G D) \Omega(G D)=\prod_{\substack{P \in \mathcal{P} \\ P \mid G D}}\left(1+\frac{1}{1+2|P|}\right)^{-1}\left(1+\frac{1}{1+2|P|}\right)^{-1} .
$$

By (3.28) and (3.29), $\Omega(G D) \leq 1 \leq \lambda_{1 / 4}(G D) \leq 2^{\omega(G D)}=2^{r+\omega(D)}$, which yields (4.28).

Proposition 4.5. We have

$$
\left|Z_{3}\right| \leq \beta_{4}(R) 2^{\tau+r+f_{G} / 2+2 \omega(D)+f_{D} / 4+M / 4} N^{1 / 2} q^{M+3 N / 4}(M+1)
$$

with $\beta_{4}(R)$ a constant.

Proof. Interchanging the order of summation in $Z_{3}$ given by (4.24) we get

$$
Z_{3}=\sum_{t \subset T} \sum_{a \in \mathcal{A}} 2^{-\omega(J(a))} \sum_{\substack{E \in \mathcal{I} \\ E \mid D}} \sum_{\substack{A \in \mathcal{I} \\ A \mid J(a) \\(1) \neq E A \neq D J(a)}} \phi_{E, A}(a)
$$

with

$$
\phi_{E, A}(a)=\sum_{t \subset T} \sum_{\begin{array}{c}
B \in \mathcal{S F}_{G D J(a)} \\
f_{B} \leq N
\end{array}} \sum_{\substack{b \in a \\
(a, b) \in \mathcal{Y} \\
B \mid J(b)}} 2^{-\omega(J(b))}(a, b)_{t \cup \Sigma(E A B)} .
$$

Let $j$ be an integer such that

$$
j<N \text {. }
$$

We divide the sum $\phi_{E, A}(a)$ into two parts according as the ideals $B$ occurring in it satisfy $f_{B} \leq j$ or $f_{B}>j$. We get

$$
\phi_{E, A}(a)=\sigma_{E, A}(a, j)+\tau_{E, A}(a, j)
$$

with

$$
\begin{aligned}
\sigma_{E, A}(a, j) & =\sum_{t \subset T} \sum_{\substack{B \in \mathcal{S F}_{G D J(a)} \\
f_{B} \leq j}} \sum_{\substack{b \in a \mathcal{Y} \\
(a, b) \in \mathcal{W} \\
B \mid J(b)}} 2^{-\omega(J(b))}(a, b)_{t \cup \Sigma(E A B)}, \\
\tau_{E, A}(a, j) & =\sum_{t \subset T} \sum_{\substack{B \in \mathcal{S}_{\mathcal{F}^{G D J(a)}} \\
f_{B}>j}} \sum_{\substack{b \in a \mathcal{Y} \\
(a, b) \in \mathcal{W} \\
B \mid J(b)}} 2^{-\omega(J(b))}(a, b)_{t \cup \Sigma(E A B)} .
\end{aligned}
$$


In (6) we set $D=E E^{\prime}, J(a)=A A^{\prime}, J(b)=B B^{\prime}$ and for $t \subset T$, let $t^{\prime}=T-t$. Then, by (4.17),

$$
\begin{aligned}
(a, b)_{t \cup \Sigma(E A B)} & =(a, b)_{t \cup \Sigma(E A B)}\left((a, b)_{t^{\prime} \cup \Sigma\left(E^{\prime} A^{\prime} B^{\prime}\right)}\right)^{2} \\
& =(a, b)_{t^{\prime} \cup \Sigma\left(E^{\prime} A^{\prime} B^{\prime}\right)} \cdot(a, b)_{T \cup \Sigma(D J(a) J(b))} \\
& =(a, b)_{t^{\prime} \cup \Sigma\left(E^{\prime} A^{\prime} B^{\prime}\right)} \cdot(a, b)_{W(a, b)} .
\end{aligned}
$$

In view of (4.19),

$$
(a, b)_{t \cup \Sigma(E A B)}=(a, b)_{t^{\prime} \cup \Sigma\left(E^{\prime} A^{\prime} B^{\prime}\right)} .
$$

Hence,

$$
\tau_{E, A}(a, j)=\sum_{t^{\prime} \subset T} \sum_{\substack{B^{\prime} \in \mathcal{S} \mathcal{F}_{G D J(a)} \\ f_{B^{\prime}}<N-j}} \sum_{\substack{b \in a \\(a, b) \in \mathcal{Y} \\ B^{\prime} \mid J(b)}} 2^{-\omega(J(b))}(a, b)_{t^{\prime} \cup \Sigma\left(E^{\prime} A^{\prime} B^{\prime}\right)} .
$$

By (5),

$$
\tau_{E, A}(a, j)=\sigma_{E^{\prime}, A^{\prime}}(a, N-j-1) .
$$

We now deal with the sum $\sigma_{E, A}(a, j)$. If $P \in \mathcal{P}(R)$ divides $D$, then $v_{P}(a)$ and $v_{P}(b)$ are odd and by (4.15), $(a, b)_{v_{P}}=\theta_{v_{P}}\left(-\operatorname{sgn}_{v_{P}}(a) \operatorname{sgn}_{v_{P}}(b)\right)$. If $P \in$ $\mathcal{P}(R)$ divides $J(a)$, then $v_{P}(a)$ is odd, $v_{P}(b)$ is even and by $(4.15),(a, b)_{v_{P}}$ $=\theta_{v_{P}}\left(\operatorname{sgn}_{v_{P}}(b)\right)$. Hence, with notations (4.13) and (4.17), $(a, b)_{\Sigma(E A)}=$ $\Theta_{\Sigma(E)}(-a b) \Theta_{\Sigma(A)}(b)$ and by symmetry, $(a, b)_{\Sigma(B)}=\Theta_{\Sigma(B)}(a)$. As in the proof of Proposition 4.3 we get

$$
\begin{aligned}
\sigma_{E, A}(a, j)= & \Theta_{E}(-a) \sum_{t_{0,1} \subset T_{0,1}} \Theta_{t_{0,1}}(a) \sum_{\substack{B \in \mathcal{S F}_{G D J(a)} \\
f_{B} \leq j}} \Theta_{\Sigma(B)}(a) \\
& \times \sum_{\substack{t_{1,0} \subset T_{1,0} \\
t_{1,1} \subset T_{1,1} \\
\left(\emptyset, t_{1,0}, t_{1,1}, A E\right) \equiv 0}} \Theta_{t_{1,1}}(a) \sum_{\substack{b \in \in_{a} \mathcal{Y} \\
B \mid J(b)}} 2^{-\omega(J(b))} \Theta_{t_{1,0} \cup t_{1,1} \cup \Sigma(A E)}(b) .
\end{aligned}
$$

Hence,

$$
\left|\sigma_{E, A}(a, j)\right| \leq \sum_{\substack{\left.t_{0,1} \subset T_{0,1} \\ t_{1,0} \subset T_{1,0} \\ t_{1,1} \subset T_{1,1} \\ \emptyset, t_{1,0}, t_{1,1}, A E\right) \equiv 0}} \sum_{\substack{B \in \mathcal{S F}_{G D J(a)} \\ f_{B} \leq j}}\left|\sum_{\substack{b \in a \mathcal{Y} \\ B \mid J(b)}} 2^{-\omega(J(b))} \Theta_{t_{1,0} \cup t_{1,1} \cup \Sigma(A E)}(b)\right| .
$$

By the parity condition, the map

$$
y \mapsto \Theta_{t_{1,0} \cup t_{1,1} \cup \Sigma(A E)}(y)
$$


is trivial on the group $k^{\star}$. As in the proof of Proposition 4.3, we get

$$
\begin{aligned}
& \left|\sum_{\substack{b \in a \mathcal{Y} \\
B \mid J(b)}} 2^{-\omega(J(b))} \Theta_{t_{1,0} \cup t_{1,1} \cup \Sigma(A E)}(b)\right| \\
& \quad \leq(q-1) \alpha_{1}(R) 2^{r+\left(f_{G}+f_{A E}\right) / 2} 2^{\omega(D J(a))} N^{1 / 2} q^{\left(N-f_{B}\right) / 2} .
\end{aligned}
$$

Hence,

$$
\begin{gathered}
\left|\sigma_{E, A}(a, j)\right| \leq(q-1) \alpha_{1}(R) 2^{r+\left(f_{G}+f_{A E}\right) / 2} 2^{\omega(D J(a))} N^{1 / 2} q^{N / 2} \\
\times \sum_{\substack{t_{0,1} \subset T_{0,1} \\
t_{1,0} \subset T_{1,0} \\
t_{1,1} \subset T_{1,1} \\
\left(\emptyset, t_{1,0}, t_{1,1}, A E\right) \equiv 0}} \sum_{\substack{B \in \mathcal{S F}_{G D J(a)} \\
f_{B} \leq j}} q^{-f_{B} / 2} \\
\end{gathered}
$$

and

$$
\begin{aligned}
\left|\sigma_{E, A}(a, j)\right| \leq & (q-1) \alpha_{1}(R) 2^{\tau+r+\left(f_{G}+f_{A E}\right) / 2} \\
& \times 2^{\omega(D J(a))} N^{1 / 2} q^{N / 2} \sum_{\substack{B \in \mathcal{S F}_{G D J(a)} \\
f_{B} \leq j}} q^{-f_{B} / 2} .
\end{aligned}
$$

By (3.5),

(9) $\left|\sigma_{E, A}(a, j)\right| \leq(q-1) h \varrho(R) \alpha_{1}(R)$

$$
\times \frac{\sqrt{q}}{\sqrt{q}-1} 2^{\tau+r+\left(f_{G}+f_{A E}\right) / 2} 2^{\omega(D J(a))} N^{1 / 2} q^{(N+j) / 2} .
$$

Similarly,

$$
\begin{aligned}
& \left|\sigma_{E^{\prime}, A^{\prime}}(a, N-j-1)\right| \\
\leq & (q-1) h \varrho(R) \alpha_{1}(R) \frac{\sqrt{q}}{\sqrt{q}-1} 2^{\tau+r+\left(f_{G}+f_{A^{\prime} E^{\prime}}\right) / 2} 2^{\omega(D J(a))} N^{1 / 2} q^{N-(j+1) / 2} .
\end{aligned}
$$

Let

$$
j=\left[\frac{1}{2}\left(N+\left(f_{E^{\prime} A^{\prime}}-f_{E A}\right) \log _{q}(2)\right)\right],
$$

where $[x]$ denotes the integral part of the real number $x$. Then, by (9) and (10),

$$
\begin{aligned}
\left|\sigma_{E, A}(a, j)\right| \leq & (q-1) h \varrho(R) \alpha_{1}(R) \\
& \times \frac{\sqrt{q}}{\sqrt{q}-1} 2^{\tau+r+f_{G} / 2} 2^{f_{D J(a)} / 4} 2^{\omega(D J(a))} N^{1 / 2} q^{3 N / 4}, \\
\left|\sigma_{E^{\prime}, A^{\prime}}(a, N-j-1)\right| \leq & (q-1) h \varrho(R) \alpha_{1}(R) \\
& \times \frac{\sqrt{q}}{\sqrt{q}-1} 2^{\tau+r+f_{G} / 2} 2^{f_{D J(a)} / 4} 2^{\omega(D J(a))} N^{1 / 2} q^{3 N / 4},
\end{aligned}
$$


and with (1), (4) and (7),

$$
\begin{aligned}
\left|Z_{3}\right| \leq & 2(q-1) h \varrho(R) \alpha_{1}(R) \\
& \times \frac{\sqrt{q}}{\sqrt{q}-1} 2^{\tau+r+f_{G} / 2+\omega(D)+f_{D} / 4} 2^{M / 4} N^{1 / 2} q^{3 N / 4} Z_{3}^{\star},
\end{aligned}
$$

where

$$
Z_{3}^{\star}=\sum_{\substack{E \in \mathcal{S} \mathcal{F} \\ E \mid D}} \sum_{a \in \mathcal{A}} \sum_{\substack{A \in \mathcal{S} \mathcal{F} \\ A \mid J(a) \\(1) \neq E A \neq D J(a)}} 1 .
$$

Interchanging the order of summation, we get

$$
Z_{3}^{\star}=(q-1) \sum_{\substack{E \in \mathcal{S} \mathcal{F} \\
E \mid D}} \sum_{\substack{A \in \mathcal{S F}_{G D} \\
f_{A} \leq M \\
(1) \neq E A \neq D J(a)}} \sum_{\begin{array}{c}
A^{\prime} \in \mathcal{S} \mathcal{F}_{A G D} \\
\mathfrak{A} U^{2} A A^{\prime} \in \operatorname{Pr} \\
f_{A A^{\prime}}=M
\end{array}} 1 .
$$

Hence, by (3.5),

$$
Z_{3}^{\star} \leq(q-1) \varrho(R) q^{M} \sum_{\substack{E \in \mathcal{S} \mathcal{F} \\ E \mid D}} \sum_{\substack{A \in \mathcal{S} \mathcal{F}_{G D} \\ f_{A} \leq M \\(1) \neq E}} q^{-f_{A}},
$$

and by (3.5) and (12),

$$
\begin{aligned}
\left|Z_{3}\right| \leq & 2(q-1)^{2} h^{2} \varrho(R)^{3} \alpha_{1}(R) \\
& \times \frac{\sqrt{q}}{\sqrt{q}-1} 2^{\tau+r+f_{G} / 2+2 \omega(D)+f_{D} / 4+M / 4} N^{1 / 2} q^{M+3 N / 4}(M+1) .
\end{aligned}
$$

This gives (4.31).

We summarize what has been proved above in the following theorem.

Theorem 4.6. Let $\theta \in] \log 2 / \log q, 1\left[\right.$. Let $\mathbf{m}=\left(m_{v}\right)_{v \in S}$ and $\mathbf{n}=$ $\left(n_{v}\right)_{v \in S}$ be $r$-tuples of rational integers such that

$$
\|\mathbf{m}\|-2 f_{U} \leq\left(\|\mathbf{n}\|-2 f_{V}-f_{D}\right) \frac{\log q}{\log 2}
$$

and

$$
\|\mathbf{m}\|-2 f_{U}-f_{D} \geq \theta\left(\|\mathbf{n}\|-2 f_{V}-f_{D}\right)>0 .
$$

Then

$$
\begin{array}{r}
\left|Z(S, \mathbf{m}, \mathbf{n}, D, U, V)-C_{1}(S) 2^{-\tau(\mathbf{m}, \mathbf{n})-\omega(D)} \Lambda(D) \frac{q^{M+N}}{\sqrt{M N}}\right| \\
\leq \beta_{5}(R, \theta) 2^{r+f_{G} / 2} 2^{\omega(D)+f_{D} / 4} \frac{q^{M+N}}{M N}
\end{array}
$$


with

$$
C_{1}(S)=\frac{2 h(q-1)}{\pi q^{g-1} \zeta_{K}\left(q^{-2}\right)} \prod_{v \in S}\left(1+\frac{1}{q^{f_{v}}}\right)^{-1},
$$

$M=\|\mathbf{m}\|-2 f_{U}-f_{D}, N=\|\mathbf{n}\|-2 f_{V}-f_{D}$, and $\beta_{5}(R, \theta)$ a constant.

Proof. By (4.21), (4.25), (4.26), (4.28) and (4.31),

$$
\begin{aligned}
\mid 2^{\omega(D)} Z-2^{1-\tau}(q-1)^{2} B_{3}(R) \Lambda(G D) & \frac{q^{M+N}}{\sqrt{M N}} \mid \\
& \leq \beta_{5}(R, \theta) 2^{r+f_{G} / 2+\omega(D)+f_{D} / 4} \frac{q^{M+N}}{M N}
\end{aligned}
$$

with $\beta_{5}(R, \theta)$ a constant. This gives (4.33) with

$$
C_{1}(S)=2(q-1)^{2} B_{3}(R) \Lambda(G) .
$$

Easy computations yield (4.34).

Corollary 4.7. Let $\theta \in] \log 2 / \log q, 1]$. Let $\mathbf{m}=\left(m_{v}\right)_{v \in S}$ and $\mathbf{n}=$ $\left(n_{v}\right)_{v \in S}$ be $r$-tuples of rational integers such that $0<\theta \max (\mathbf{m}, \mathbf{n}) \leq$ $\min (\mathbf{m}, \mathbf{n})$. Then

$$
\left|H_{1}(S, \mathbf{m}, \mathbf{n})-C_{1}(S) 2^{-\tau(\mathbf{m}, \mathbf{n})} \frac{q^{\|\mathbf{m}\|+\|\mathbf{n}\|}}{\sqrt{\|\mathbf{m}\|\|\mathbf{n}\|}}\right| \leq \beta_{5}(R, \theta) 2^{r+f_{G} / 2} \frac{q^{\|\mathbf{m}\|+\|\mathbf{n}\|}}{\|\mathbf{m}\|\|\mathbf{n}\|} .
$$

Proof. Interchanging $\mathbf{m}$ and $\mathbf{n}$ if necessary, we apply Theorem 4.6 with $D=U=V=1$.

For ideals $U$ and $V$ of $R$ coprime to $G$ and such that $2 f_{U} \leq\|\mathbf{m}\|$ and $2 f_{V} \leq\|\mathbf{n}\|$, let $\mathcal{Y}^{\prime}=\mathcal{Y}^{\prime}(S, \mathbf{m}, \mathbf{n}, U, V)$ denote the set of $(a, b) \in \mathcal{X}(S, \mathbf{m}, \mathbf{n})$ such that $U(a)=U$ and $U(b)=V$, let $\mathcal{Z}^{\prime}(S, \mathbf{m}, \mathbf{n}, U, V)$ denote the set of $(a, b) \in \mathcal{Y}^{\prime}(S, \mathbf{m}, \mathbf{n}, U, V)$ such that the quadratic form $\left(f_{a, b}\right)$ represents 0 over $K$, and let $Z^{\prime}=Z^{\prime}(S, \mathbf{m}, \mathbf{n}, U, V)=\# \mathcal{Z}^{\prime}(S, \mathbf{m}, \mathbf{n}, D, U, V)$.

Fix ideals $U$ and $V$ of $R$ coprime to $G$. The following theorem gives an estimate for the numbers $Z^{\prime}(S, \mathbf{m}, \mathbf{n}, U, V)$.

Theorem 4.8. Let $\alpha \in] \log 2 / \log q, 1]$. Let $\mathbf{m}=\left(m_{v}\right)_{v \in S}$ and $\mathbf{n}=$ $\left(n_{v}\right)_{v \in S}$ be $r$-tuples of rational integers such that $2 f_{U} \leq\|\mathbf{m}\|, 2 f_{V} \leq\|\mathbf{n}\|$ and

$$
\min \left(\|\mathbf{m}\|-2 f_{U},\|\mathbf{n}\|-2 f_{V}\right) \geq \alpha \max \left(\|\mathbf{m}\|-2 f_{U},\|\mathbf{n}\|-2 f_{V}\right)>0 .
$$

Then

$$
\begin{aligned}
\mid Z^{\prime}(S, \mathbf{m}, \mathbf{n}, U, V) & -2^{-\tau(\mathbf{m}, \mathbf{n})} C^{\prime}(S) \frac{q^{\|\mathbf{m}\|+\|\mathbf{n}\|-2 f_{U}-2 f_{V}}}{\sqrt{\left(\|\mathbf{m}\|-2 f_{U}\right)\left(\|\mathbf{n}\|-2 f_{V}\right)}} \mid \\
& \leq \beta_{6}(R, \alpha) 2^{r+f_{G} / 2} \frac{q^{\|\mathbf{m}\|+\|\mathbf{n}\|-2 f_{U}-2 f_{V}}}{\left(\|\mathbf{m}\|-2 f_{U}\right)\left(\|\mathbf{n}\|-2 f_{V}\right)}
\end{aligned}
$$


with

$$
C^{\prime}(S)=\frac{2 h(q-1)}{\pi q^{g-1} \zeta_{K}\left(q^{-2}\right)} \cdot \prod_{\substack{v \in V \\ v \notin S}}\left(1+\frac{1}{2 q^{f_{v}}\left(1+q^{f_{v}}\right)}\right) \cdot \prod_{v \in S}\left(1+\frac{1}{q^{f_{v}}}\right)^{-1}
$$

and $\beta_{6}(R, \alpha)$ a constant.

Proof. The set $\mathcal{Z}^{\prime}(S, \mathbf{m}, \mathbf{n}, U, V)$ is the union of the sets $\mathcal{Z}(S, \mathbf{m}, \mathbf{n}$, $D, U, V)$ for $D$ running over the set of square-free ideals of $R$ coprime to $G$. Hence

$$
Z^{\prime}(S, \mathbf{m}, \mathbf{n}, U, V)=\sum_{\substack{D \in \mathcal{S} \mathcal{F}_{G} \\ f_{D} \leq m}} Z(S, \mathbf{m}, \mathbf{n}, D, U, V)
$$

with

$$
m=\min \left(\|\mathbf{m}\|-2 f_{U},\|\mathbf{n}\|-2 f_{V}\right)
$$

Let

$$
M^{\prime}=\|\mathbf{m}\|-2 f_{U}, \quad N^{\prime}=\|\mathbf{n}\|-2 f_{V} .
$$

By symmetry, we may suppose

$$
M^{\prime} \leq N^{\prime}
$$

Condition (4.36) gives

$$
M^{\prime} \geq \alpha N^{\prime}
$$

Obviously,

$$
Z(S, \mathbf{m}, \mathbf{n}, D, U, V) \leq(q-1)^{2} \sum_{\substack{A \in \mathcal{S} \mathcal{F} \\ \mathfrak{A} A \in \operatorname{Pr} \\ f_{A D}=M^{\prime}}} \sum_{\substack{B \in \mathcal{S} \mathcal{F} \\ \mathfrak{B} B \in \operatorname{Pr} \\ f_{B D}=N^{\prime}}} 1
$$

and by (3.5),

$$
Z(S, \mathbf{m}, \mathbf{n}, D, U, V) \leq(q-1)^{2} \varrho(R)^{2} q^{M^{\prime}+N^{\prime}-2 f_{D}} .
$$

Let

$$
\kappa(\alpha)=\kappa=\frac{\alpha-\log 2 / \log q}{\alpha(2-\alpha-\log 2 / \log q)} \quad \text { and } \quad \theta=\alpha \frac{1-\kappa}{1-\kappa \alpha}
$$

Then

$$
\theta=\frac{1}{2}\left(\alpha+\frac{\log 2}{\log q}\right)>\frac{\log 2}{\log q} \text { and } \quad \alpha=\frac{\theta}{1-\kappa(1-\theta)}
$$

Hence,

$$
M^{\prime}-\theta N^{\prime} \geq \kappa M^{\prime}(1-\theta) .
$$

Let

$$
\mu=\left[\kappa M^{\prime}\right],
$$




$$
Z^{\star}=\sum_{\substack{D \in \mathcal{S} \mathcal{F}_{G} \\ f_{D} \leq \mu}} Z(S, \mathbf{m}, \mathbf{n}, D, U, V)
$$

Then, by (1),

$$
0 \leq Z^{\prime}-Z^{\star} \leq(q-1)^{2} \varrho(R)^{2} q^{M^{\prime}+N^{\prime}} \sum_{\substack{D \in \mathcal{S F}_{G} \\ f_{D}>\mu}} q^{-2 f_{D}},
$$

and by (3.5),

$$
0 \leq Z^{\prime}-Z^{\star} \leq h(q-1) \varrho(R)^{3} q^{M^{\prime}+N^{\prime}-\mu} .
$$

By (6) and (7), each $D$ occurring in the sum $Z^{\star}$ satisfies conditions (4.11) and (4.32). In view of Theorem 4.6,

$$
\begin{aligned}
\mid Z(S, \mathbf{m}, \mathbf{n}, D, U, V)- & 2^{-\tau(\mathbf{m}, \mathbf{n})-\omega(D)} C_{1}(S) \Lambda(D) \frac{q^{M^{\prime}+N^{\prime}-2 f_{D}}}{\sqrt{\left(M^{\prime}-f_{D}\right)\left(N^{\prime}-f_{D}\right)}} \mid \\
& \leq \beta_{5}(R, \theta) 2^{r+f_{G} / 2} 2^{\omega(D)+f_{D} / 4} \frac{q^{M^{\prime}+N^{\prime}-2 f_{D}}}{\left(M^{\prime}-f_{D}\right)\left(N^{\prime}-f_{D}\right)}
\end{aligned}
$$

By (7),

$$
\frac{q^{M^{\prime}+N^{\prime}-2 f_{D}}}{\left(M^{\prime}-f_{D}\right)\left(N^{\prime}-f_{D}\right)} \leq\left(\frac{1}{1-\kappa}\right)^{2} \frac{q^{M^{\prime}+N^{\prime}-2 f_{D}}}{M^{\prime} N^{\prime}} .
$$

Hence,

$$
\begin{aligned}
& \mid Z(S, \mathbf{m}, \mathbf{n}, D, U, V) \\
& \quad-C_{1}(S) 2^{-\tau(\mathbf{m}, \mathbf{n})-\omega(D)} \Lambda(D) \frac{q^{M^{\prime}+N^{\prime}-2 f_{D}}}{\sqrt{\left(M^{\prime}-f_{D}\right)\left(N^{\prime}-f_{D}\right)}} \mid \\
& \quad \leq\left(\frac{1}{1-\kappa}\right)^{2} \beta_{5}(R, \lambda) 2^{r+f_{G} / 2} 2^{\omega(D)+f_{D} / 4} \frac{q^{M^{\prime}+N^{\prime}}}{M^{\prime} N^{\prime}}
\end{aligned}
$$

For any ideal $D$,

$$
2^{\omega(D)+f_{D} / 4} \leq q^{f_{D} \log _{q}(2) / 4} \prod_{\substack{P \in \mathcal{P} \\ P \mid D}} q^{f_{P} \log _{q}(2)} \leq|D|^{5 \log _{q}(2) / 4} .
$$

Hence, in view of (3.5), the series

$$
Y_{1}=\sum_{D \in \mathcal{S} \mathcal{F}} 2^{\omega(D)+f_{D} / 4} q^{-2 f_{D}}
$$

is convergent. By (7), (8), (10) and (11),

$$
\begin{aligned}
\left|Z^{\star}-C_{1}(S) 2^{-\tau(\mathbf{m}, \mathbf{n})} q^{M^{\prime}+N^{\prime}} Z^{\star \star}\right| & \\
& \leq 2^{r+f_{G} / 2}\left(\frac{1}{1-\kappa}\right)^{2} Y_{1} \beta_{5}(R, \theta) \frac{q^{M^{\prime}+N^{\prime}}}{M^{\prime} N^{\prime}},
\end{aligned}
$$


where

$$
Z^{\star \star}=\sum_{\substack{D \in \mathcal{S F}_{G} \\ f_{D} \leq \mu}} \frac{2^{-\omega(D)} q^{-2 f_{D}} \Lambda(D)}{\sqrt{\left(M^{\prime}-f_{D}\right)\left(N^{\prime}-f_{D}\right)}} .
$$

By (6), if the ideal $D$ is such that $f_{D} \leq \mu$ then

$$
0 \leq \frac{1}{\sqrt{\left(M^{\prime}-f_{D}\right)\left(N^{\prime}-f_{D}\right)}}-\frac{1}{\sqrt{M^{\prime} N^{\prime}}} \leq \gamma(\alpha) \frac{f_{D}}{M^{\prime} N^{\prime}}
$$

with

$$
\gamma(\alpha)=\frac{1}{\sqrt{1-\kappa(\alpha)}+1-\kappa(\alpha)}\left(\frac{1}{\sqrt{1-\kappa(\alpha)}}+\frac{1}{\sqrt{\alpha}}\right)
$$

and by (13),

$$
\begin{aligned}
0 & \leq Z^{\star \star}-\frac{1}{\sqrt{M^{\prime} N^{\prime}}} \sum_{\substack{D \in \mathcal{S F}_{G} \\
f_{D} \leq \mu}} 2^{-\omega(D)} q^{-2 f_{D}} \Lambda(D) \\
& \leq \frac{\gamma(\alpha)}{M^{\prime} N^{\prime}} \sum_{\substack{D \in \mathcal{S F}_{G} \\
f_{D} \leq \mu}} f_{D} 2^{-\omega(D)} q^{-2 f_{D}} \Lambda(D) .
\end{aligned}
$$

By $(4.30), 0<\Lambda(D) \leq 1$ for all $D \in \mathcal{I}$. Hence, by (3.5), the series

$$
Y_{2}=\sum_{D \in \mathcal{S F}_{G}} f_{D} 2^{-\omega(D)} q^{-2 f_{D}} \Lambda(D)
$$

is convergent and

$$
0 \leq Z^{\star \star}-\frac{1}{\sqrt{M^{\prime} N^{\prime}}} \sum_{\substack{D \in \mathcal{S F}_{G} \\ f_{D} \leq \mu}} 2^{-\omega(D)} q^{-2 f_{D}} \Lambda(D) \leq \frac{\gamma(\alpha) Y_{2}}{M^{\prime} N^{\prime}} .
$$

The series

$$
Y_{3}=\sum_{D \in \mathcal{S F}_{G}} 2^{-\omega(D)} q^{-2 f_{D}} \Lambda(D)
$$

is convergent and in view of (4.30),

$$
Y_{3}=\prod_{\substack{v \in V \\ v \notin S}}\left(1+\frac{1}{2 q^{f_{v}}\left(q^{f_{v}}+1\right)}\right)
$$

By (4.30) and (3.5),

$$
\sum_{\substack{D \in \mathcal{S F}_{G} \\ f_{D}>\mu}} 2^{-\omega(D)} q^{-2 f_{D}} \Lambda(D) \leq \sum_{\substack{D \in \mathcal{I} \\ f_{D}>\mu}} q^{-2 f_{D}} \leq \frac{h \varrho(R)}{q-1} q^{-\mu}
$$


Hence by (17),

$$
\left|Z^{\star \star}-\frac{Y_{3}}{\sqrt{M^{\prime} N^{\prime}}}\right| \leq \frac{h \varrho(R)}{q-1} \cdot \frac{q^{-\mu}}{\sqrt{M^{\prime} N^{\prime}}}+\frac{\gamma(\alpha) Y_{2}}{M^{\prime} N^{\prime}}
$$

and by (9) and (12),

$$
\begin{aligned}
\mid Z^{\prime}- & C_{1}(S) Y_{3} 2^{-\tau(\mathbf{m}, \mathbf{n})} \frac{q^{M^{\prime}+N^{\prime}}}{\sqrt{M^{\prime} N^{\prime}}} \mid \\
\leq & h(q-1) \varrho(R)^{3} q^{M^{\prime}+N^{\prime}-\mu}+2^{r+f_{G} / 2}\left(\frac{1}{1-\kappa}\right)^{2} Y_{1} \beta_{5}(R, \theta) \frac{q^{M^{\prime}+N^{\prime}}}{M^{\prime} N^{\prime}} \\
& +\frac{h C_{1}(S) \varrho(R) q^{M^{\prime}+N^{\prime}-\mu}}{(q-1) \sqrt{M^{\prime} N^{\prime}}}+\frac{C_{1}(S) \gamma(\alpha) Y_{2}}{M^{\prime} N^{\prime}} .
\end{aligned}
$$

Hence,

$$
\left|Z^{\prime}-C_{1}(S) Y_{3} 2^{-\tau(\mathbf{m}, \mathbf{n})} \frac{q^{M^{\prime}+N^{\prime}}}{\sqrt{M^{\prime} N^{\prime}}}\right| \leq \beta_{6}(S, \alpha) \frac{q^{M^{\prime}+N^{\prime}}}{M^{\prime} N^{\prime}}
$$

with $\beta_{6}(S, \alpha)$ a constant. In view of $(3),(20)$ gives $(4.37)$ with $C^{\prime}(S)=$ $Y_{3} C_{1}(S)$. We get (4.38) from (19) and (4.34).

Corollary 4.9. Let $\alpha \in] \log 2 / \log q, 1]$. Let $\mathbf{m}=\left(m_{v}\right)_{v \in S}$ and $\mathbf{n}=$ $\left(n_{v}\right)_{v \in S}$ be $r$-tuples of rational integers such that $\min (\|\mathbf{m}\|,\|\mathbf{n}\|) \geq$ $\alpha \max (\|\mathbf{m}\|,\|\mathbf{n}\|)>0$. Then

$$
\left|H^{\prime}(\mathbf{m}, \mathbf{n})-2^{-\tau(\mathbf{m}, \mathbf{n})} C^{\prime}(S) \frac{q^{\|\mathbf{m}\|+\|\mathbf{n}\|}}{\sqrt{\|\mathbf{m}\|\|\mathbf{n}\|}}\right| \leq \beta_{6}(R, \alpha) \frac{q^{\|\mathbf{m}\|+\|\mathbf{n}\|}}{\|\mathbf{m}\|\|\mathbf{n}\|} .
$$

Proof. Take $U=V=(1)$ in Theorem 4.8.

Now, we are able to end the proof.

Theorem 4.10. Let $\lambda \in] 3 \log 2 /(2 \log q), 1]$. Let $\mathbf{m}=\left(m_{v}\right)_{v \in S}$ and $\mathbf{n}=\left(n_{v}\right)_{v \in S}$ be r-tuples of rational integers such that $\min (\|\mathbf{m}\|,\|\mathbf{n}\|) \geq$ $\lambda \max (\|\mathbf{m}\|,\|\mathbf{n}\|)>0$. Then

$$
\left|H(\mathbf{m}, \mathbf{n})-2^{-\tau(\mathbf{m}, \mathbf{n})} C(S) \frac{q^{\|\mathbf{m}\|+\|\mathbf{n}\|}}{\sqrt{\|\mathbf{m}\|\|\mathbf{n}\|}}\right| \leq \beta_{7}(S, \lambda) \frac{q^{\|\mathbf{m}\|+\|\mathbf{n}\|}}{\|\mathbf{m}\|\|\mathbf{n}\|}
$$

with

$$
\begin{aligned}
C(S)= & \frac{2 h \zeta_{K}\left(q^{-2}\right)(q-1)}{\pi q^{g-1}} \\
& \times \prod_{v \in S}\left(1-\frac{1}{q^{2 f_{v}}}\right)\left(1-\frac{1}{q^{f_{v}}}\right) \cdot \prod_{\substack{v \in V \\
v \notin S}}\left(1+\frac{1}{2 q^{f_{v}}\left(q^{f_{v}}+1\right)}\right)
\end{aligned}
$$

and $\beta_{7}(S, \lambda)$ a constant. 
Proof. We suppose that $\|\mathbf{m}\| \leq\|\mathbf{n}\|$. Then

$$
\|\mathbf{m}\| \geq \lambda\|\mathbf{n}\| \text {. }
$$

In view of (4.5) and the definition of $Z^{\prime}(S, \mathbf{m}, \mathbf{n}, U, V)$,

$$
H(S, \mathbf{m}, \mathbf{n})=\sum_{\substack{U \in \mathcal{I}_{G} \\ 2 f_{U} \leq\|\mathbf{m}\|}} \sum_{\substack{V \in \mathcal{I}_{G} \\ 2 f_{V} \leq\|\mathbf{n}\|}} Z^{\prime}(S, \mathbf{m}, \mathbf{n}, U, V) .
$$

Let

$$
\alpha=\frac{2}{3} \lambda \text {. }
$$

We note that $\log 2 / \log q<\alpha \leq 2 / 3$. Let $E^{\prime}$ denote the set of pairs $(U, V)$ with $U$ and $V$ coprime to $G$ and such that $2 f_{U} \leq \frac{1}{3}\|\mathbf{m}\|, 2 f_{V} \leq \frac{1}{3} \lambda\|\mathbf{n}\|$, and let $E$ denote the set of $(U, V) \in E^{\prime}$ such that

$$
\min \left(\|\mathbf{m}\|-2 f_{U},\|\mathbf{n}\|-2 f_{V}\right) \geq \alpha \max \left(\|\mathbf{m}\|-2 f_{U},\|\mathbf{n}\|-2 f_{V}\right) .
$$

Let $(U, V)$ be a pair of ideals as in (2). Obviously, $Z^{\prime}(S, \mathbf{m}, \mathbf{n}, U, V)$ is less than the number of pairs $(A, B)$ of ideals such that $A \mathfrak{A}$ and $B \mathfrak{B}$ are principal and satisfy

$$
f_{A}=\|\mathbf{m}\|-2 f_{U}, \quad f_{B}=\|\mathbf{n}\|-2 f_{V},
$$

with $\mathfrak{A}$ and $\mathfrak{B}$ defined by (4.7). By (3.5),

$$
Z^{\prime}(S, \mathbf{m}, \mathbf{n}, U, V) \leq \varrho(R)^{2} q^{\|\mathbf{m}\|+\|\mathbf{n}\|-2 f_{U}-2 f_{V}} .
$$

Hence,

$$
\begin{aligned}
& \sum_{\substack{U \in \mathcal{I}_{\mathcal{G}}, V \in \mathcal{I}_{G} \\
(U, V) \notin E^{\prime} \\
2 f_{U} \leq\|\mathbf{m}\|, 2 f_{V} \leq\|\mathbf{n}\|}} Z^{\prime}(S, \mathbf{m}, \mathbf{n}, U, V) \\
& \leq \varrho(R)^{2} q^{\|\mathbf{m}\|+\|\mathbf{n}\|}\left(\sum_{\substack{U \in \mathcal{I} \\
2 f_{U}>\|\mathbf{m}\| / 3}} q^{-2 f_{U}} \sum_{V \in \mathcal{I}} q^{-2 f_{V}}+\sum_{\substack{V \in \mathcal{I} \\
2 f_{V}>\lambda\|\mathbf{n}\| / 3}} q^{-2 f_{V}} \sum_{U \in \mathcal{I}} q^{-2 f_{U}}\right) .
\end{aligned}
$$

Thus, by (3.5),

$$
\begin{aligned}
& \sum_{\substack{U \in \mathcal{I}_{\mathcal{G}}, V \in \mathcal{I}_{G} \\
(U, V) \notin E^{\prime} \\
2 f_{U} \leq\|\mathbf{m}\|, 2 f_{V} \leq\|\mathbf{n}\|}} Z^{\prime}(S, \mathbf{m}, \mathbf{n}, U, V) \\
& \quad \leq h^{2}\left(\frac{q}{q-1}\right)^{2} \varrho(R)^{4} q^{\|\mathbf{m}\|+\|\mathbf{n}\|}\left(q^{-\|\mathbf{m}\| / 6}+q^{-\lambda\|\mathbf{n}\| / 6}\right),
\end{aligned}
$$

and by (1), 
(5)

$$
\begin{aligned}
& \sum_{\substack{U \in \mathcal{I}_{\mathcal{G}}, V \in \mathcal{I}_{G} \\
(U, V) \notin E^{\prime} \\
2 f_{U} \leq\|\mathbf{m}\|, 2 f_{V} \leq\|\mathbf{n}\|}} Z^{\prime}(S, \mathbf{m}, \mathbf{n}, U, V) \\
& \quad \leq 2 h^{2}\left(\frac{q}{q-1}\right)^{2} \varrho(R)^{4} q^{\|\mathbf{m}\|+\|\mathbf{n}\|-\lambda\|\mathbf{n}\| / 6} .
\end{aligned}
$$

If $(U, V) \in E^{\prime}$ is not in $E$ then either $2 f_{V} \geq\|\mathbf{n}\|-\|\mathbf{m}\|+2 f_{U}$, and in this case $2 f_{V}>\|\mathbf{n}\|-\alpha\|\mathbf{m}\|+2 \alpha f_{U} \geq\|\mathbf{n}\|-\alpha\|\mathbf{m}\|$, or $2 f_{V}<\|\mathbf{n}\|-\|\mathbf{m}\|+2 f_{U}$, and in this case $2 f_{U}>\|\mathbf{m}\|-\alpha\|\mathbf{n}\|+2 \alpha f_{V} \geq\|\mathbf{m}\|-\alpha\|\mathbf{n}\|$. So by (3.5),

$$
\begin{aligned}
& \sum_{\substack{(U, V) \in E^{\prime} \\
(U, V) \notin E}} Z^{\prime}(S, \mathbf{m}, \mathbf{n}, U, V) \\
& \quad \leq h^{2}\left(\frac{q}{q-1}\right)^{2} \varrho(R)^{4} q^{\|\mathbf{m}\|+\|\mathbf{n}\|}\left(q^{-(\|\mathbf{n}\|-\alpha\|\mathbf{m}\|) / 2}+q^{-(\|\mathbf{m}\|-\alpha\|\mathbf{n}\|) / 2}\right) .
\end{aligned}
$$

Let

$$
F=F(S, \mathbf{m}, \mathbf{n})=\sum_{(U, V) \in E} Z^{\prime}(S, \mathbf{m}, \mathbf{n}, U, V) .
$$

Then, by (2), (5), (1) and (3),

$$
|H(S, \mathbf{m}, \mathbf{n})-F| \leq 4 h^{2}\left(\frac{q}{q-1}\right)^{2} \varrho(R)^{4} q^{\|\mathbf{m}\|+\|\mathbf{n}\|-\lambda\|\mathbf{n}\| / 6} .
$$

If $(U, V) \in E$, then

$$
\min \left(\|\mathbf{m}\|-2 f_{U},\|\mathbf{n}\|-2 f_{V}\right) \geq \alpha \max \left(\|\mathbf{m}\|-2 f_{U},\|\mathbf{n}\|-2 f_{V}\right)
$$

with $\log 2 / \log q<\alpha \leq 1$ and we may apply Theorem 4.8 to $Z^{\prime}(S, \mathbf{m}, \mathbf{n}, U, V)$. Doing this, we get

$$
\left|F-2^{-\tau(\mathbf{m}, \mathbf{n})} C^{\prime}(S) q^{\|\mathbf{m}\|+\|\mathbf{n}\|} F^{\star}\right| \leq \beta_{6}(S, \varrho) 2^{r+f_{G} / 2} q^{\|\mathbf{m}\|+\|\mathbf{n}\|} F^{\prime}
$$

with

$$
\begin{aligned}
F^{\star} & =\sum_{(U, V) \in E} q^{-2 f_{U}-2 f_{V}}\left(\|\mathbf{m}\|-2 f_{U}\right)^{-1 / 2}\left(\|\mathbf{n}\|-2 f_{V}\right)^{-1 / 2}, \\
F^{\prime} & =\sum_{(U, V) \in E} q^{-2 f_{U}-2 f_{V}}\left(\|\mathbf{m}\|-2 f_{U}\right)^{-1}\left(\|\mathbf{n}\|-2 f_{V}\right)^{-1} .
\end{aligned}
$$

If $(U, V) \in E$ then $(U, V) \in E^{\prime}$ and by $(3),\left(\|\mathbf{m}\|-2 f_{U}\right)\left(\|\mathbf{n}\|-2 f_{V}\right) \geq$ $\frac{2}{9}(3-\lambda)\|\mathbf{m}\|\|\mathbf{n}\|$. Therefore,

$$
F^{\prime} \leq \frac{9}{2(3-\lambda)\|\mathbf{m}\|\|\mathbf{n}\|}\left(\sum_{U \in \mathcal{I}} q^{-2 f_{U}}\right)^{2} .
$$

By (3.5),

$$
F^{\prime} \leq \frac{9 h^{2} q^{2} \varrho(R)^{2}}{2(3-\lambda)(q-1)^{2}\|\mathbf{m}\|\|\mathbf{n}\|}
$$


We have

$$
\begin{aligned}
0 \leq & \frac{1}{\sqrt{\left(\|\mathbf{m}\|-2 f_{U}\right)\left(\|\mathbf{n}\|-2 f_{V}\right)}}-\frac{1}{\sqrt{\|\mathbf{m}\|\|\mathbf{n}\|}} \\
\leq & \frac{2 f_{V}}{\sqrt{2 / 3}(\sqrt{1-\lambda / 3}+1-\lambda / 3)\|\mathbf{m}\|^{1 / 2}\|\mathbf{n}\|^{3 / 2}} \\
& +\frac{2 f_{U}}{(\sqrt{2 / 3}+2 / 3)\|\mathbf{m}\|^{3 / 2}\|\mathbf{n}\|^{1 / 2}} \\
\leq & \frac{1}{\|\mathbf{m}\|\|\mathbf{n}\|}\left(\frac{2 f_{V}}{\sqrt{2 / 3}(\sqrt{1-\lambda / 3}+1-\lambda / 3)}+\frac{2 f_{U}}{\sqrt{\lambda}(\sqrt{2 / 3}+2 / 3)}\right) .
\end{aligned}
$$

The series

$$
Y_{4}=\sum_{(U, V) \in \mathcal{I} \times \mathcal{I}} f_{U} q^{-2 f_{U}-2 f_{V}}
$$

is convergent and by (10),

$$
0 \leq F^{\star}-\frac{1}{\sqrt{\|\mathbf{m}\|\|\mathbf{n}\|}} \sum_{(U, V) \in E} q^{-2 f_{U}-2 f_{V}} \leq \frac{\kappa(\lambda) Y_{4}}{\|\mathbf{m}\|\|\mathbf{n}\|}
$$

with

$$
\kappa(\lambda)=2\left(\frac{1}{\sqrt{2 / 3}(\sqrt{1-\lambda / 3}+1-\lambda / 3)}+\frac{1}{\sqrt{\lambda}(\sqrt{2 / 3}+2 / 3)}\right) .
$$

The series

$$
Y_{5}=\sum_{(U, V) \in \mathcal{I}_{G} \times \mathcal{I}_{G}} q^{-2 f_{U}-2 f_{V}}
$$

is convergent. As above we get

$$
\left|Y_{5}-\sum_{(U, V) \in E} q^{-2 f_{U}-2 f_{V}}\right| \leq 4 h^{2}\left(\frac{q}{q-1}\right)^{2} \varrho(R)^{2} q^{\|\mathbf{m}\|+\|\mathbf{n}\|-\lambda\|\mathbf{n}\| / 6},
$$

and by (14),

$$
\begin{aligned}
0 & \leq F^{\star}-\frac{Y_{5}}{\sqrt{\|\mathbf{m}\|\|\mathbf{n}\|}} \\
& \leq \frac{\kappa(\lambda) Y_{4}}{\|\mathbf{m}\|\|\mathbf{n}\|}+4 h^{2}\left(\frac{q}{q-1}\right)^{2} \varrho(R)^{2} \frac{q^{\|\mathbf{m}\|+\|\mathbf{n}\|-\lambda\|\mathbf{n}\| / 6}}{\sqrt{\|\mathbf{m}\|\|\mathbf{n}\|}} .
\end{aligned}
$$

By (8), (9), (12) and (17) we get

$$
\left|H(S, \mathbf{m}, \mathbf{n})-2^{-\tau(\mathbf{m}, \mathbf{n})} C^{\prime}(S) Y_{5} \frac{q^{\|\mathbf{m}\|+\|\mathbf{n}\|}}{\sqrt{\|\mathbf{m}\|\|\mathbf{n}\|}}\right| \leq \beta_{7}(R, \lambda) \frac{q^{\|\mathbf{m}\|+\|\mathbf{n}\|}}{\|\mathbf{m}\|\|\mathbf{n}\|}
$$


with $\beta_{7}(R, \lambda)$ a constant. In order to complete the proof, it remains to compute the constant

$$
C(S)=C^{\prime}(S) Y_{5} .
$$

Expanding $Y_{5}$ as a product, we get

$$
Y_{5}=\prod_{P \in \mathcal{P}_{G}}\left(1-q^{-2 f_{P}}\right)^{-2}=\prod_{P \in \mathcal{P}}\left(1-q^{-2 f_{P}}\right)^{-2} \cdot \prod_{\substack{P \in \mathcal{P} \\ P \mid G}}\left(1-q^{-2 f_{P}}\right)^{2} .
$$

By (2.1) and (4.1),

$$
Y_{5}=\zeta_{K}\left(\frac{1}{q^{2}}\right)^{2} \prod_{v \in S}\left(1-\frac{1}{q^{2 f_{v}}}\right)^{2}
$$

and by (4.38),

$$
\begin{aligned}
C(S)= & \frac{2 h \zeta_{K}\left(q^{-2}\right)(q-1)}{\pi q^{g-1}} \cdot \prod_{\substack{v \in V \\
v \notin S}}\left(1+\frac{1}{2 q^{f_{v}}\left(q^{f_{v}}+1\right)}\right) \\
& \times \prod_{v \in S}\left(1-\frac{1}{q^{2 f_{v}}}\right)^{-2}\left(1+\frac{1}{q^{f_{v}}}\right)^{-1} \cdot
\end{aligned}
$$

5. Quadratic forms with coefficients in the ring $R_{S}$. In this section we end the proof of the announced theorem.

Let $S$ be a finite, non-empty set of $r$ places of $K$. For $r$-tuples $\mathbf{m}=$ $\left(m_{v}\right)_{v \in S}$ and $\mathbf{n}=\left(n_{v}\right)_{v \in S}$ of rational integers, let $Q_{S}(\mathbf{m}, \mathbf{n})$ denote the number of $(a, b) \in R_{S} \times R_{S}$ such that

(1) $v(a)=m_{v}$ and $v(b)=n_{v}$ for all $v \in S$,

(2) the quadratic form

$$
X^{2}-a Y^{2}-b Z^{2}
$$

represents 0 over the field $K$.

Similarly, let $Q_{1, S}(\mathbf{m}, \mathbf{n})$ denote the number of $(a, b) \in R_{S} \times R_{S}$ with ideals $R_{S} a$ and $R_{S} b$ square-free and coprime and such that (1) and (2) are true; and let $Q_{S}^{\prime}(\mathbf{m}, \mathbf{n})$ denote the number of $(a, b) \in R_{S} \times R_{S}$ with $R_{S} a$ and $R_{S} b$ square-free and such that (1) and (2) are true.

TheOrem 5.1. Let $\lambda$ and $\theta$ be real numbers with $3 \log 2 /(2 \log q)<\lambda \leq 1$ and $\log 2 / \log q<\theta \leq 1$. Let $\mathbf{m}=\left(m_{v}\right)_{v \in S}$ and $\mathbf{n}=\left(n_{v}\right)_{v \in S}$ be $r$-tuples of rational integers. If

$$
0<\lambda \max (\|\mathbf{m}\|,\|\mathbf{n}\|) \leq \min (\|\mathbf{m}\|,\|\mathbf{n}\|),
$$


then

$$
\left|Q_{S}(\mathbf{m}, \mathbf{n})-2^{-\tau(\mathbf{m}, \mathbf{n})} C(S) \frac{q^{\|\mathbf{m}\|+\|\mathbf{n}\|}}{\sqrt{\|\mathbf{m}\|\|\mathbf{n}\|}}\right| \leq \beta_{7}(S, \lambda) \frac{q^{\|\mathbf{m}\|+\|\mathbf{n}\|}}{\|\mathbf{m}\|\|\mathbf{n}\|}
$$

if

$$
0<\theta \max (\|\mathbf{m}\|,\|\mathbf{n}\|) \leq \min (\|\mathbf{m}\|,\|\mathbf{n}\|)
$$

then

$$
\begin{gathered}
\left|Q_{1, S}(\mathbf{m}, \mathbf{n})-2^{-\tau(\mathbf{m}, \mathbf{n})} C_{1}(S) \frac{q^{\|\mathbf{m}\|+\|\mathbf{n}\|}}{\sqrt{\|\mathbf{m}\|\|\mathbf{n}\|}}\right| \leq \beta_{5}(S, \theta) \frac{q^{\|\mathbf{m}\|+\|\mathbf{n}\|}}{\|\mathbf{m}\|\|\mathbf{n}\|}, \\
\left|Q_{S}^{\prime}(\mathbf{m}, \mathbf{n})-2^{-\tau(\mathbf{m}, \mathbf{n})} C^{\prime}(S) \frac{q^{\|\mathbf{m}\|+\|\mathbf{n}\|}}{\sqrt{\|\mathbf{m}\|\|\mathbf{n}\|}}\right| \leq \beta_{6}(S, \theta) \frac{q^{\|\mathbf{m}\|+\|\mathbf{n}\|}}{\|\mathbf{m}\|\|\mathbf{n}\|},
\end{gathered}
$$

with $\|\cdot\|, \tau(\mathbf{m}, \mathbf{n}), C(S), C^{\prime}(S), C_{1}(S), \beta_{5}(S, \theta), \beta_{6}(S, \theta), \beta_{7}(S, \lambda)$ defined as in Section 4.

Proof. Let $v_{0} \in S$ and let $R=R_{\left\{v_{0}\right\}}$. If $S=\left\{v_{0}\right\}$, then (5.2), (5.4) and (5.5) are respectively given by Theorem 4.10, Corollary 4.7 and Corollary 4.9. We now assume that the set $S^{\prime}=S-\left\{v_{0}\right\}$ is not empty. Let $G$ be defined by (4.1). For $v \in S^{\prime}$, we denote by $d_{v}$ the order of the ideal class of $P_{v}$ in the ideal class group of the ring $R$, and by $p_{v}$ the monic element such that $R p_{v}=P_{v}^{d_{v}}$. Let $\left(i_{v}\right)_{v \in S^{\prime}},\left(\bar{m}_{v}\right)_{v \in S^{\prime}},\left(j_{v}\right)_{v \in S^{\prime}},\left(\bar{n}_{v}\right)_{v \in S^{\prime}}$ be defined by the relations

$$
m_{v}=2 d_{v} i_{v}+\bar{m}_{v}, 0 \leq \bar{m}_{v}<2 d_{v} ; \quad n_{v}=2 d_{v} j_{v}+\bar{n}_{v}, 0 \leq \bar{n}_{v}<2 d_{v} .
$$

Let $\mathbf{X}_{S}(\mathbf{m}, \mathbf{n})$ denote the set of $(a, b) \in R_{S} \times R_{S}$ such that $\mathbf{v}(a)=\mathbf{m}$ and $\mathbf{v}(b)=\mathbf{n}$. Let $(a, b) \in \mathbf{X}_{S}(\mathbf{m}, \mathbf{n})$ and set

$$
a^{\prime}=a \prod_{v \in S^{\prime}} p_{v}^{-2 i_{v}}, \quad b^{\prime}=b \prod_{v \in S^{\prime}} p_{v}^{-2 j_{v}} .
$$

We look at $\left(v\left(a^{\prime}\right)\right)_{v \in V}$ and $\left(v\left(b^{\prime}\right)\right)_{v \in V}$. For $v \in S^{\prime}$, we have $v\left(a^{\prime}\right)=\bar{m}_{v} \geq 0$ and $v\left(b^{\prime}\right)=\bar{n}_{v} \geq 0$. For $v \notin S, v\left(a^{\prime}\right)=v(a) \geq 0$ and $v\left(b^{\prime}\right)=v(b) \geq 0$. Hence $a^{\prime}$ and $b^{\prime}$ belong to the ring $R=R_{\left\{v_{0}\right\}}$. By the product formula,

$$
v_{0}\left(a^{\prime}\right)=m_{v_{0}}+\frac{2}{f_{0}} \sum_{v \in S^{\prime}} i_{v} d_{v} f_{v}, \quad v_{0}\left(b^{\prime}\right)=n_{v_{0}}+\frac{2}{f_{0}} \sum_{v \in S^{\prime}} j_{v} d_{v} f_{v} .
$$

Hence, $\left(a^{\prime}, b^{\prime}\right) \in \mathcal{X}_{S}\left(\mathbf{m}^{\prime}, \mathbf{n}^{\prime}\right)$, where

$$
\mathbf{m}^{\prime}=\left(\bar{m}_{v}\right)_{v \in S}, \quad \mathbf{n}^{\prime}=\left(\bar{n}_{v}\right)_{v \in S},
$$

with

$$
\bar{m}_{v_{0}}=m_{v_{0}}+\frac{2}{f_{0}} \sum_{v \in S^{\prime}} i_{v} d_{v} f_{v}, \quad \bar{n}_{v_{0}}=n_{v_{0}}+\frac{2}{f_{0}} \sum_{v \in S^{\prime}} j_{v} d_{v} f_{v} .
$$

Moreover, the map $(a, b) \mapsto\left(a^{\prime}, b^{\prime}\right)$ is bijective and the quadratic form $\left(f_{a, b}\right)$ represents 0 over $K$ if and only if $\left(f_{a^{\prime}, b^{\prime}}\right)$ does. Hence, $Q_{S}(\mathbf{m}, \mathbf{n})$ is equal 
to the number of pairs $\left(a^{\prime}, b^{\prime}\right) \in \mathcal{X}_{S}\left(\mathbf{m}^{\prime}, \mathbf{n}^{\prime}\right)$ such that $\left(f_{a^{\prime}, b^{\prime}}\right)$ represents 0 over $K$, that is, the number $H\left(S, \mathbf{m}^{\prime}, \mathbf{n}^{\prime}\right)$ defined in the previous section. By (3)-(5),

$$
-\left\|\mathbf{m}^{\prime}\right\|=\sum_{v \in S} f_{v} \bar{m}_{v}=f_{0} m_{v_{0}}+\sum_{v \in S^{\prime}} 2 i_{v} d_{v} f_{v}+\sum_{v \in S^{\prime}}\left(m_{v}-2 i_{v} d_{v}\right) f_{v},
$$

and

$$
\left\|\mathbf{m}^{\prime}\right\|=\|\mathbf{m}\|, \quad\left\|\mathbf{n}^{\prime}\right\|=\|\mathbf{n}\| .
$$

We now deduce (5.2) from (4.40).

Let $\mathbf{X}_{1, S}(\mathbf{m}, \mathbf{n})$, resp. $\mathbf{X}_{S}^{\prime}(\mathbf{m}, \mathbf{n})$, denote the set of $(a, b) \in \mathbf{X}_{S}(\mathbf{m}, \mathbf{n})$ such that $\left(f_{a, b}\right)$ represents zero with $a$ and $b$ coprime and square-free, resp. with $a$ and $b$ square-free. As above, the map $(a, b) \mapsto\left(a^{\prime}, b^{\prime}\right)$ is bijective from $\mathbf{X}_{1, S}(\mathbf{m}, \mathbf{n})$ to $\mathcal{Z}\left(S, \mathbf{m}^{\prime}, \mathbf{n}^{\prime},(1),(1),(1)\right)$, and from $\mathbf{X}_{S}^{\prime}(\mathbf{m}, \mathbf{n})$ to $\mathcal{Z}\left(S, \mathbf{m}^{\prime}, \mathbf{n}^{\prime},(1),(1)\right)$, the sets $\mathcal{Z}\left(S, \mathbf{m}^{\prime}, \mathbf{n}^{\prime},(1),(1),(1)\right)$ and $\mathcal{Z}^{\prime}\left(S, \mathbf{m}^{\prime}, \mathbf{n}^{\prime}\right.$, $(1),(1))$ being defined in Section 4. We now get (5.4) and (5.5) from (4.35) and (4.39).

\section{References}

[1] M. Car, Quadratic forms with polynomial coefficients, Acta Arith. 113 (2004), 131155.

[2] - Classes modulo les puissances dans l'anneau des $S$-entiers d'un corps de fonctions, ibid. 118 (2005), 149-185.

[3] M. Deuring, Lectures on the Theory of Algebraic Functions of One Variable, Lecture Notes in Math. 314, Springer, 1973.

[4] C. R. Guo, On solvability of ternary quadratic forms, Proc. London Math. Soc. (3) 70 (1995), 241-263.

[5] C. Hooley, On ternary quadratic forms that represent zero, Glasgow Math. J. 35 (1993), 13-23.

[6] R. E. MacRae, On unique factorization in certain rings of algebraic functions, J. Algebra 17 (1971), 243-261.

[7] J.-P. Serre, Corps locaux, Act. Sci. Industr. 1296, Hermann, Paris, 1962.

[8] - Spécialisation des éléments de $\mathrm{Br}_{2}\left(\mathbb{Q}\left(T_{1}, \ldots, T_{n}\right)\right)$, C. R. Acad. Sci. Paris Sér. I Math. 311 (1990), 397-402.

[9] A. Weil, Basic Number Theory, 3rd ed., Grundlehren Math. Wiss. 144, Springer, 1974.

LATP, Faculté des Sciences et Techniques

Université Paul Cézanne-Aix-Marseille III

Case cour A

Avenue Escadrille Normandie-Niemen

F-13397 Marseille Cedex 20, France

E-mail: mireille.car@univ-cezanne.fr 